\title{
Applying GG-Convex Function to Hermite-Hadamard Inequalities Involving Hadamard Fractional Integrals
}

\author{
Zhi Zhang, ${ }^{1}$ JinRong Wang, ${ }^{1,2}$ and JianHua Deng ${ }^{1}$ \\ ${ }^{1}$ Department of Mathematics, Guizhou University, Guiyang, Guizhou 550025, China \\ ${ }^{2}$ School of Mathematics and Computer Science, Guizhou Normal College, Guiyang, Guizhou 550018, China \\ Correspondence should be addressed to JinRong Wang; wjr9668@126.com
}

Received 1 February 2014; Revised 7 June 2014; Accepted 10 June 2014; Published 14 July 2014

Academic Editor: Hari M. Srivastava

Copyright (C) 2014 Zhi Zhang et al. This is an open access article distributed under the Creative Commons Attribution License, which permits unrestricted use, distribution, and reproduction in any medium, provided the original work is properly cited.

By virtue of fractional integral identities, incomplete beta function, useful series, and inequalities, we apply the concept of GG-convex function to derive new type Hermite-Hadamard inequalities involving Hadamard fractional integrals. Finally, some applications to special means of real numbers are demonstrated.

\section{Introduction}

Fractional calculus played an important role in various fields such as electricity, biology, economics, and signal and image processing [1-8]. The fractional Hermite-Hadamard inequality gives a lower and an upper estimation for both righthand and left-hand integrals average of any convex function defined on a compact interval, involving the midpoint and the endpoints of the domain.

As we know, Set [9] firstly studied fractional Ostrowski inequalities involving Riemann-Liouville fractional integrals. Then, Sarikaya et al. [10] studied Hermite-Hadamard type inequalities involving Riemann-Liouville fractional integrals. Further, our group go on studying fractional version Hermite-Hadamard inequality involving Riemann-Liouville and Hadamard fractional integrals for all kinds of functions [11-19].

Recently, Wang et al. $[16,17]$ established the following two powerful fractional integral identities involving Hadamard fractional integrals.

Lemma 1 (see [12, Lemma 3.1]). Let $f:[a, b] \rightarrow \mathbb{R}$ be a differentiable mapping on $(a, b)$. If $f^{\prime} \in L[a, b]$, then the following equality for fractional integrals holds:

$$
\begin{array}{r}
\frac{f(a)+f(b)}{2}-\frac{\Gamma(\alpha+1)}{2(\ln b-\ln a)^{\alpha}}\left[{ }_{H} J_{a^{+}}^{\alpha} f(b)+{ }_{H} J_{b^{-}}^{\alpha} f(a)\right] \\
=\frac{\ln b-\ln a}{2} \int_{0}^{1}\left[(1-t)^{\alpha}-t^{\alpha}\right] a^{t} b^{1-t} f^{\prime}\left(a^{t} b^{1-t}\right) d t
\end{array}
$$

where the symbols ${ }_{H} J_{a^{+}}^{\alpha} f$ and ${ }_{H} J_{b^{-}}^{\alpha} f$ are defined by

$$
\begin{array}{r}
\left({ }_{H} J_{a^{+}}^{\alpha} f\right)(x)=\frac{1}{\Gamma(\alpha)} \int_{a}^{x}\left(\ln \frac{x}{t}\right)^{\alpha-1} f(t) \frac{d t}{t}, \\
(0<a<x \leq b), \\
\left({ }_{H} J_{b^{-}}^{\alpha} f\right)(x)=\frac{1}{\Gamma(\alpha)} \int_{x}^{b}\left(\ln \frac{t}{x}\right)^{\alpha-1} f(t) \frac{d t}{t}, \\
(0<a \leq x<b),
\end{array}
$$

where $\Gamma(\cdot)$ is the Gamma function.

Lemma 2 (see [11, Lemma 2.1]). Let $f:[a, b] \rightarrow \mathbb{R}$ be $a$ differentiable mapping on $(a, b)$ with $0<a<b$. If $f^{\prime} \in L[a, b]$, then the following equality for fractional integrals holds: 


$$
\begin{aligned}
& \frac{\Gamma(\alpha+1)}{2(\ln b-\ln a)^{\alpha}}\left[{ }_{H} J_{a^{+}}^{\alpha} f(b)+{ }_{H} J_{b^{-}}^{\alpha} f(a)\right]-f\left(\frac{a+b}{2}\right) \\
& \quad=\frac{b-a}{2} \int_{0}^{1} k f^{\prime}(t a+(1-t) b) d t \\
& \quad-\frac{\ln b-\ln a}{2} \int_{0}^{1}\left[(1-t)^{\alpha}-t^{\alpha}\right] a^{t} b^{1-t} f^{\prime}\left(a^{t} b^{1-t}\right) d t
\end{aligned}
$$

where

$$
k= \begin{cases}1, & 0 \leq t<\frac{1}{2} \\ -1, & \frac{1}{2} \leq t<1 .\end{cases}
$$

Remark 3. It is remarkable that Professor Srivastava et al. [20] give some further refinements and extensions of the HermiteHadamard inequalities in $n$ variables. In the forthcoming works, we will try to extend to study fractional version Hermite-Hadamard inequalities in $n$ variables based on such fundamental results.

Next, we recall the following basic concepts and results in our previous papers.

Definition 4 (see $[21,22]$ ). Let $f: I \subseteq \mathbb{R}^{+} \rightarrow \mathbb{R}^{+}$. A function $f$ is said to be GG-convex on $I$ if, for every $x, y \in I$ and $\lambda \epsilon$ $[0,1]$, one has

$$
f\left(x^{\lambda} y^{1-\lambda}\right) \leq[f(x)]^{\lambda}[f(y)]^{1-\lambda} .
$$

Remark 5. By the arithmetic-geometric mean inequality, we have

$$
[f(x)]^{\lambda}[f(y)]^{1-\lambda} \leq \lambda f(x)+(1-\lambda) f(y)
$$

Linking (5) and (6), we obtain

$$
f\left(x^{\lambda} y^{1-\lambda}\right) \leq \lambda f(x)+(1-\lambda) f(y)
$$

which appears in the standard definition of GA-convex function [21]. So GG-convex function is GA-convex function.

Lemma 6 (see $[19$, Lemma 2.5]). For $t \in[0,1], x, y>0$, one has

$$
t x+(1-t) y \geq y^{1-t} x^{t}
$$

Lemma 7 (see [13, Lemma 2.1]). For $\alpha>0$ and $k>0$, one has

$$
I(\alpha)=\int_{0}^{1} t^{\alpha-1} k^{t} d t=k \sum_{i=1}^{\infty}(-1)^{i-1} \frac{(\ln k)^{i-1}}{(\alpha)_{i}}<+\infty,
$$

where $(\alpha)_{i}=\alpha(\alpha+1)(\alpha+2) \cdots(\alpha+i-1)$.

Lemma 8 (see [13, Lemma 2.2]). For $\alpha>0$ and $k>0, z>0$, one has

$$
\begin{gathered}
J(\alpha, k)=\int_{0}^{1}(1-t)^{\alpha-1} k^{t} d t=\sum_{i=1}^{\infty} \frac{(\ln k)^{i-1}}{(\alpha)_{i}}<+\infty, \\
H(\alpha, k, z)=\int_{0}^{z} t^{\alpha-1} k^{t} d t=z^{\alpha} k^{z} \sum_{i=1}^{\infty} \frac{(-z \ln k)^{i-1}}{(\alpha)_{i}}<+\infty .
\end{gathered}
$$

Lemma 9 (see [13, Lemma 2.3]). For $\alpha>0$ and $k>0,1>$ $z>0$, one has

$$
\begin{aligned}
R(\alpha, k, z) & =\int_{0}^{z}(1-t)^{\alpha-1} k^{t} d t \\
& =\sum_{i=0}^{\infty} \frac{(\ln k)^{i-1}}{(\alpha)_{i}}\left(1-k^{z}(1-z)^{\alpha+i-1}\right) .
\end{aligned}
$$

In the present paper, we will use the above concepts and lemmas to derive some new fractional Hermite-Hadamard inequalities involving Hadamard fractional integrals.

\section{Main Results Based on Lemma 1}

Now we are ready to state the following main results in this section.

Theorem 10. Let $f:[0, b] \rightarrow \mathbb{R}^{+}$be a differentiable mapping. If $\left|f^{\prime}\right|$ is measurable and $\left|f^{\prime}\right|$ is GG-convex on $[a, b]$ for some fixed $\alpha \in(0, \infty)$ and $t \in[0,1], 0 \leq a<b$, then the following integrals hold:

$$
\begin{aligned}
& \left|\frac{f(a)+f(b)}{2}-\frac{\Gamma(\alpha+1)}{2(\ln b-\ln a)^{\alpha}}\left[{ }_{H} J_{a^{+}}^{\alpha} f(b)+{ }_{H} J_{b^{-}}^{\alpha} f(a)\right]\right| \\
& \leq \frac{\ln b-\ln a}{2} \\
& \times\left\{\left(1-\frac{1}{2^{\alpha+1}}\right)\right. \\
& \times\left[\frac{b\left|f^{\prime}(b)\right|}{\alpha+1}+\frac{b\left|f^{\prime}(a)\right|+a\left|f^{\prime}(b)\right|-2 b\left|f^{\prime}(b)\right|}{\alpha+2}\right] \\
& +\frac{a\left|f^{\prime}(a)\right|-a\left|f^{\prime}(b)\right|-b\left|f^{\prime}(a)\right|}{\alpha+3} \\
& -\frac{a\left|f^{\prime}(a)\right|-b\left|f^{\prime}(a)\right|}{(\alpha+3) 2^{\alpha+3}}+\frac{2 a\left|f^{\prime}(a)\right|}{(\alpha+1)(\alpha+2)(\alpha+3)} \\
& +\frac{b\left|f^{\prime}(a)\right|+a\left|f^{\prime}(b)\right|-2 a\left|f^{\prime}(a)\right|}{(\alpha+1)(\alpha+3)} \\
& +\frac{2 a\left(\left|f^{\prime}(a)\right|-\left|f^{\prime}(b)\right|\right)-2 b\left|f^{\prime}(a)\right|}{2^{\alpha+3}(\alpha+2)(\alpha+3)} \\
& +\frac{b\left|f^{\prime}(a)\right|+a\left|f^{\prime}(b)\right|}{2^{\alpha+2}(\alpha+2)} \\
& \left.+\frac{a\left|f^{\prime}(a)\right|}{(\alpha+1) 2^{\alpha+2}}-\frac{a\left|f^{\prime}(a)\right|}{(\alpha+2)^{2} 2^{\alpha+2}}\right\} \text {. }
\end{aligned}
$$


Proof. Noting Definition 4 and Lemmas 1 and 6, we have

$$
\begin{aligned}
& \left|\frac{f(a)+f(b)}{2}-\frac{\Gamma(\alpha+1)}{2(\ln b-\ln a)^{\alpha}}\left[{ }_{H} J_{a^{+}}^{\alpha} f(b)+{ }_{H} J_{b^{-}}^{\alpha} f(a)\right]\right| \\
& =\left|\frac{\ln b-\ln a}{2} \int_{0}^{1} a^{t} b^{1-t}\left[(1-t)^{\alpha}-t^{\alpha}\right] f^{\prime}\left(a^{t} b^{1-t}\right) d t\right| \\
& \leq \frac{\ln b-\ln a}{2} \int_{0}^{1} a^{t} b^{1-t}\left|(1-t)^{\alpha}-t^{\alpha}\right|\left|f^{\prime}\left(a^{t} b^{1-t}\right)\right| d t \\
& \leq \frac{\ln b-\ln a}{2} \int_{1 / 2}^{1} a^{t} b^{1-t}\left[t^{\alpha}-(1-t)^{\alpha}\right]\left|f^{\prime}\left(a^{t} b^{1-t}\right)\right| d t \\
& +\frac{\ln b-\ln a}{2} \int_{0}^{1 / 2} a^{t} b^{1-t}\left[(1-t)^{\alpha}-t^{\alpha}\right] \\
& \times\left|f^{\prime}\left(a^{t} b^{1-t}\right)\right| d t \\
& \leq \frac{\ln b-\ln a}{2} \int_{1 / 2}^{1}[a t+b(1-t)]\left[t^{\alpha}-(1-t)^{\alpha}\right] \\
& \times\left|f^{\prime}(a)\right|^{t}\left|f^{\prime}(b)\right|^{1-t} d t \\
& +\frac{\ln b-\ln a}{2} \int_{0}^{1 / 2}[a t+b(1-t)]\left[(1-t)^{\alpha}-t^{\alpha}\right] \\
& \times\left|f^{\prime}(a)\right|^{t}\left|f^{\prime}(b)\right|^{1-t} d t \\
& \leq \frac{\ln b-\ln a}{2} \int_{1 / 2}^{1}[a t+b(1-t)]\left[t^{\alpha}-(1-t)^{\alpha}\right] \\
& \times\left[\left|f^{\prime}(a)\right| t+\left|f^{\prime}(b)\right|(1-t)\right] d t \\
& +\frac{\ln b-\ln a}{2} \int_{0}^{1 / 2}[a t+b(1-t)]\left[(1-t)^{\alpha}-t^{\alpha}\right] \\
& \times\left[\left|f^{\prime}(a)\right| t+\left|f^{\prime}(b)\right|(1-t)\right] d t \\
& \leq \frac{\ln b-\ln a}{2}\left\{\frac{a\left|f^{\prime}(a)\right|}{\alpha+3} \int_{1 / 2}^{1}(\alpha+3) t^{\alpha+2} d t\right. \\
& +\frac{b\left|f^{\prime}(a)\right|}{\alpha+2} \int_{1 / 2}^{1}(\alpha+2) t^{\alpha+1} d t \\
& -\frac{b\left|f^{\prime}(a)\right|}{\alpha+3} \int_{1 / 2}^{1}(\alpha+3) t^{\alpha+2} d t \\
& +\frac{a\left|f^{\prime}(b)\right|}{\alpha+2} \int_{1 / 2}^{1}(\alpha+2) t^{\alpha+1} d t \\
& -\frac{a\left|f^{\prime}(b)\right|}{\alpha+3} \int_{1 / 2}^{1}(\alpha+3) t^{\alpha+2} d t \\
& +\frac{b\left|f^{\prime}(b)\right|}{\alpha+1} \int_{1 / 2}^{1}(\alpha+1) t^{\alpha} d t \\
& -\frac{2 b\left|f^{\prime}(b)\right|}{\alpha+2} \int_{1 / 2}^{1}(\alpha+2) t^{\alpha+1} d t
\end{aligned}
$$

$$
\begin{aligned}
& +\frac{b\left|f^{\prime}(b)\right|}{\alpha+3} \int_{1 / 2}^{1}(\alpha+3) t^{\alpha+2} d t \\
& -a\left|f^{\prime}(a)\right| \int_{1 / 2}^{1}(1-t)^{\alpha} t^{2} d t \\
& -b\left|f^{\prime}(a)\right| \int_{1 / 2}^{1} t(1-t)^{\alpha+1} d t \\
& -a\left|f^{\prime}(b)\right| \int_{1 / 2}^{1} t(1-t)^{\alpha+1} d t \\
& \left.-\frac{b\left|f^{\prime}(b)\right|}{\alpha+3} \int_{1 / 2}^{1}(\alpha+3)(1-t)^{\alpha+2} d t\right\} \\
& +\frac{\ln b-\ln a}{2}\left\{a\left|f^{\prime}(a)\right| \int_{0}^{1 / 2} t^{2}(1-t)^{\alpha} d t\right. \\
& +b\left|f^{\prime}(a)\right| \int_{0}^{1 / 2} t(1-t)^{\alpha+1} d t \\
& +a\left|f^{\prime}(b)\right| \int_{0}^{1 / 2} t(1-t)^{\alpha+1} d t \\
& +\frac{b\left|f^{\prime}(b)\right|}{\alpha+3} \int_{0}^{1 / 2}(\alpha+3)(1-t)^{\alpha+2} d t \\
& -\frac{a\left|f^{\prime}(a)\right|}{\alpha+3} \int_{0}^{1 / 2}(\alpha+3) t^{\alpha+2} d t \\
& -\frac{b\left|f^{\prime}(a)\right|}{\alpha+2} \int_{0}^{1 / 2}(\alpha+2) t^{\alpha+1} d t \\
& +\frac{b\left|f^{\prime}(a)\right|}{\alpha+3} \int_{0}^{1 / 2}(\alpha+3) t^{\alpha+2} d t \\
& -\frac{a\left|f^{\prime}(b)\right|}{\alpha+2} \int_{0}^{1 / 2}(\alpha+2) t^{\alpha+1} d t \\
& +\frac{a\left|f^{\prime}(b)\right|}{\alpha+3} \int_{0}^{1 / 2}(\alpha+3) t^{\alpha+2} d t \\
& -\frac{b\left|f^{\prime}(b)\right|}{\alpha+1} \int_{0}^{1 / 2}(\alpha+1) t^{\alpha} d t \\
& +\frac{2 b\left|f^{\prime}(b)\right|}{\alpha+2} \int_{0}^{1 / 2}(\alpha+2) t^{\alpha+1} d t \\
& \left.-\frac{b\left|f^{\prime}(b)\right|}{\alpha+3} \int_{0}^{1 / 2}(\alpha+3) t^{\alpha+2} d t\right\} \\
& \leq \frac{\ln b-\ln a}{2} \\
& \times\left\{\frac{a\left|f^{\prime}(a)\right|}{\alpha+3}\left(1-\frac{1}{2^{\alpha+3}}\right)\right.
\end{aligned}
$$




$$
\begin{aligned}
& +\frac{b\left|f^{\prime}(a)\right|}{\alpha+2}\left(1-\frac{1}{2^{\alpha+2}}\right)-\frac{b\left|f^{\prime}(a)\right|}{\alpha+3}\left(1-\frac{1}{2^{\alpha+3}}\right) \\
& +a\left|f^{\prime}(a)\right| \int_{0}^{1 / 2}(1-t)^{\alpha} t^{2} d t \\
& +\frac{a\left|f^{\prime}(b)\right|}{\alpha+2}\left(1-\frac{1}{2^{\alpha+2}}\right)-\frac{a\left|f^{\prime}(b)\right|}{\alpha+3}\left(1-\frac{1}{2^{\alpha+3}}\right) \quad-b\left|f^{\prime}(a)\right| \int_{0}^{1} t(1-t)^{\alpha+1} d t \\
& +\frac{b\left|f^{\prime}(b)\right|}{\alpha+1}\left(1-\frac{1}{2^{\alpha+1}}\right)-\frac{2 b\left|f^{\prime}(b)\right|}{\alpha+2}\left(1-\frac{1}{2^{\alpha+2}}\right) \quad+b\left|f^{\prime}(a)\right| \int_{0}^{1 / 2} t(1-t)^{\alpha+1} d t \\
& +\frac{b\left|f^{\prime}(b)\right|}{\alpha+3}\left(1-\frac{1}{2^{\alpha+3}}\right)-a\left|f^{\prime}(a)\right| \int_{1 / 2}^{1}(1-t)^{\alpha} t^{2} d t \quad-a\left|f^{\prime}(b)\right| \int_{0}^{1} t(1-t)^{\alpha+1} d t \\
& -b\left|f^{\prime}(a)\right| \int_{1 / 2}^{1} t(1-t)^{\alpha+1} d t \\
& -a\left|f^{\prime}(b)\right| \int_{1 / 2}^{1} t(1-t)^{\alpha+1} d t \\
& \left.-\frac{b\left|f^{\prime}(b)\right|}{\alpha+3} \int_{1 / 2}^{1}(\alpha+3)(1-t)^{\alpha+2} d t\right\} \\
& +\frac{\ln b-\ln a}{2} \\
& \times\left\{a\left|f^{\prime}(a)\right| \int_{0}^{1 / 2} t^{2}(1-t)^{\alpha} d t\right. \\
& +b\left|f^{\prime}(a)\right| \int_{0}^{1 / 2} t(1-t)^{\alpha+1} d t \\
& +a\left|f^{\prime}(b)\right| \int_{0}^{1 / 2} t(1-t)^{\alpha+1} d t \\
& +\frac{b\left|f^{\prime}(b)\right|}{\alpha+3}\left(\frac{1}{2^{\alpha+3}}-1\right)-\frac{a\left|f^{\prime}(a)\right|}{\alpha+3} \frac{1}{2^{\alpha+3}} \\
& -\frac{b\left|f^{\prime}(a)\right|}{\alpha+2} \frac{1}{2^{\alpha+2}}+\frac{b\left|f^{\prime}(a)\right|}{\alpha+3} \frac{1}{2^{\alpha+3}} \\
& -\frac{a\left|f^{\prime}(b)\right|}{\alpha+2} \frac{1}{2^{\alpha+2}}+\frac{a\left|f^{\prime}(b)\right|}{\alpha+3} \frac{1}{2^{\alpha+3}} \\
& -\frac{b\left|f^{\prime}(b)\right|}{\alpha+1} \frac{1}{2^{\alpha+1}}+\frac{2 b\left|f^{\prime}(b)\right|}{\alpha+2} \frac{1}{2^{\alpha+2}} \\
& \left.-\frac{b\left|f^{\prime}(a)\right|}{\alpha+3} \frac{1}{2^{\alpha+3}}\right\} \\
& \leq \frac{\ln b-\ln a}{2}\left\{\frac{(a-b)\left[\left|f^{\prime}(a)\right|-\left|f^{\prime}(b)\right|\right]}{\alpha+3}\left(1-\frac{1}{2^{\alpha+3}}\right)\right. \\
& +\frac{b\left|f^{\prime}(b)\right|}{\alpha+1}\left(1-\frac{1}{2^{\alpha+1}}\right) \\
& +\frac{b\left[\left|f^{\prime}(a)\right|-\left|f^{\prime}(b)\right|\right]+(a-b)\left|f^{\prime}(b)\right|}{\alpha+2} \\
& \times\left(1-\frac{1}{2^{\alpha+2}}\right)-a\left|f^{\prime}(a)\right| \int_{0}^{1}(1-t)^{\alpha} t^{2} d t \\
& \left.+a\left|f^{\prime}(b)\right| \int_{0}^{1 / 2} t(1-t)^{\alpha+1} d t\right\} \\
& +\frac{\ln b-\ln a}{2}\left\{a\left|f^{\prime}(a)\right| \int_{0}^{1 / 2} t^{2}(1-t)^{\alpha} d t\right. \\
& +b\left|f^{\prime}(a)\right| \int_{0}^{1 / 2} t(1-t)^{\alpha+1} d t \\
& +a\left|f^{\prime}(b)\right| \int_{0}^{1 / 2} t(1-t)^{\alpha+1} d t \\
& +\frac{b\left|f^{\prime}(b)\right|}{\alpha+3}\left(\frac{1}{2^{\alpha+3}}-1\right)-\frac{a\left|f^{\prime}(b)\right|}{(\alpha+3) 2^{\alpha+3}} \\
& -\frac{b\left|f^{\prime}(a)\right|+a\left|f^{\prime}(b)\right|-2 b\left|f^{\prime}(b)\right|}{(\alpha+2) 2^{\alpha+2}} \\
& \left.-\frac{b\left|f^{\prime}(b)\right|}{(\alpha+1) 2^{\alpha+1}}\right\} \\
& \leq \frac{\ln b-\ln a}{2} \\
& \times\left\{\left(1-\frac{1}{2^{\alpha+1}}\right)\right. \\
& \times\left[\frac{b\left|f^{\prime}(b)\right|}{\alpha+1}\right. \\
& \left.+\frac{b\left|f^{\prime}(a)\right|+a\left|f^{\prime}(b)\right|-2 b\left|f^{\prime}(b)\right|}{\alpha+2}\right] \\
& +\frac{a\left|f^{\prime}(a)\right|-a\left|f^{\prime}(b)\right|-b\left|f^{\prime}(a)\right|}{\alpha+3} \\
& -\frac{a\left|f^{\prime}(a)\right|-b\left|f^{\prime}(a)\right|}{(\alpha+3) 2^{\alpha+3}} \\
& +\frac{2 a\left|f^{\prime}(a)\right|}{(\alpha+1)(\alpha+2)(\alpha+3)} \\
& +\frac{b\left|f^{\prime}(a)\right|+a\left|f^{\prime}(b)\right|-2 a\left|f^{\prime}(a)\right|}{(\alpha+1)(\alpha+3)}
\end{aligned}
$$




$$
\begin{aligned}
& +\frac{2 a\left(\left|f^{\prime}(a)\right|-\left|f^{\prime}(b)\right|\right)-2 b\left|f^{\prime}(a)\right|}{2^{\alpha+3}(\alpha+2)(\alpha+3)} \\
& +\frac{b\left|f^{\prime}(a)\right|+a\left|f^{\prime}(b)\right|}{2^{\alpha+2}(\alpha+2)}+\frac{a\left|f^{\prime}(a)\right|}{(\alpha+1) 2^{\alpha+2}} \\
& \left.-\frac{a\left|f^{\prime}(a)\right|}{(\alpha+2)^{2} 2^{\alpha+2}}\right\} .
\end{aligned}
$$

The proof is done.

Theorem 11. Let $f:[0, b] \rightarrow \mathbb{R}^{+}$be a differentiable mapping. If $\left|f^{\prime}\right|^{q}$ is measurable and $\left|f^{\prime}\right|^{q},(q>1)$ is GG-convex on $[a, b]$ for some fired $\alpha \in(0, \infty)$ and $t \in[0,1], 0 \leq a<b$, then the following integrals hold:

$$
\begin{gathered}
\left|\frac{f(a)+f(b)}{2}-\frac{\Gamma(\alpha+1)}{2(\ln b-\ln a)^{\alpha}}\left[{ }_{H} J_{a^{+}}^{\alpha} f(b)+{ }_{H} J_{b^{-}}^{\alpha} f(a)\right]\right| \\
\leq \frac{\ln b-\ln a}{2}\left(\frac{\left|f^{\prime}(a)\right|^{q}+2\left|f^{\prime}(b)\right|^{q}-\left|f^{\prime}(b)\right|^{q}}{2}\right)^{1 / q} \\
\quad \times\left(\frac{a^{p}-2 b^{p}}{p \alpha+2}+\frac{b^{p}}{p \alpha+1}-\frac{2 b^{p}+a^{p}}{2^{p \alpha+2}(p \alpha+1)}\right. \\
\quad+\frac{2 b^{p}-a^{p}}{2^{p \alpha+2}(p \alpha+2)}-\frac{a^{p}}{p \alpha(p \alpha+1)} \\
\quad-\frac{a^{p}+b^{p}}{2^{p \alpha+2}(p \alpha+1)(p \alpha+2)} \\
\left.+\frac{2 a^{p}}{(p \alpha+1)(p \alpha+2)}\right)^{1 / p},
\end{gathered}
$$

where $1 / p+1 / q=1$.

Proof. By using Definition 4 and Lemmas 1 and 6, we have

$$
\begin{aligned}
& \left|\frac{f(a)+f(b)}{2}-\frac{\Gamma(\alpha+1)}{2(\ln b-\ln a)^{\alpha}}\left[{ }_{H} J_{a^{+}}^{\alpha} f(b)+{ }_{H} J_{b^{-}}^{\alpha} f(a)\right]\right| \\
& \leq \frac{\ln b-\ln a}{2}\left(\int_{0}^{1} a^{p t} b^{p(1-t)}\left|(1-t)^{\alpha}-t^{\alpha}\right|^{p} d t\right)^{1 / p} \\
& \quad \times\left(\int_{0}^{1}\left|f^{\prime}(a)\right|^{q t}\left|f^{\prime}(b)\right|^{q(1-t)} d t\right)^{1 / q} \\
& \leq \frac{\ln b-\ln a}{2} \\
& \quad \times\left(\int_{0}^{1}\left[t a^{p}+(1-t) b^{p}\right]\left|(1-t)^{\alpha}-t^{\alpha}\right|^{p} d t\right)^{1 / p} \\
& \quad \times\left(\int_{0}^{1}\left[t\left|f^{\prime}(a)\right|^{q}+(1-t)\left|f^{\prime}(b)\right|^{q}\right] d t\right)^{1 / q}
\end{aligned}
$$

$$
\begin{aligned}
\leq & \frac{\ln b-\ln a}{2} \\
\times & \left(\int_{1 / 2}^{1}\left[t a^{p}+(1-t) b^{p}\right]\left(t^{p a}-(1-t)^{p a}\right) d t\right. \\
& \left.\quad+\int_{0}^{1 / 2}\left[t a^{p}+(1-t) b^{p}\right]\left[(1-t)^{p \alpha}-t^{p \alpha}\right] d t\right)^{1 / p} \\
& \times\left(\frac{\left|f^{\prime}(a)\right|^{q}}{2}+\left|f^{\prime}(b)\right|^{q}-\frac{\left|f^{\prime}(b)\right|^{q}}{2}\right)^{1 / q}
\end{aligned}
$$$$
\leq \frac{\ln b-\ln a}{2}\left(\frac{\left|f^{\prime}(a)\right|^{q}+2\left|f^{\prime}(b)\right|^{q}-\left|f^{\prime}(b)\right|^{q}}{2}\right)^{1 / q}
$$$$
\times\left(a^{p} \int_{1 / 2}^{1} t^{p \alpha+1} d t-a^{p} \int_{1 / 2}^{1} t(1-t)^{p \alpha} d t\right.
$$$$
+b^{p} \int_{1 / 2}^{1} t^{p \alpha}(1-t) d t-b^{p} \int_{1 / 2}^{1}(1-t)^{p \alpha+1} d t
$$$$
+a^{p} \int_{0}^{1 / 2} t(1-t)^{p \alpha} d t-a^{p} \int_{0}^{1 / 2} t^{p \alpha+1} d t
$$$$
+b^{p} \int_{0}^{1 / 2}(1-t)^{p \alpha+1} d t
$$$$
\left.-b^{p} \int_{0}^{1 / 2} t^{p \alpha}(1-t) d t\right)^{1 / p}
$$$$
\leq \frac{\ln b-\ln a}{2}\left(\frac{\left|f^{\prime}(a)\right|^{q}+2\left|f^{\prime}(b)\right|^{q}-\left|f^{\prime}(b)\right|^{q}}{2}\right)^{1 / q}
$$$$
\times\left(\frac{a^{p}}{p \alpha+2}-a^{p} \int_{0}^{1} t(1-t)^{p \alpha-1} d t\right.
$$$$
+a^{p} \int_{0}^{1 / 2} t(1-t)^{p \alpha} d t+\frac{b^{p}}{p \alpha+1}
$$$$
-\frac{1}{2^{p \alpha+1}} \frac{b^{p}}{p \alpha+1}-\frac{b^{p}}{p \alpha+2}+\frac{1}{2^{p \alpha+2}} \frac{b^{p}}{p \alpha+2}
$$$$
+a^{p} \int_{0}^{1 / 2} t(1-t)^{p \alpha} d t-\frac{1}{2^{p \alpha+2}} \frac{a^{p}}{p \alpha+2}
$$$$
+\frac{1}{2^{p \alpha+2}} \frac{b^{p}}{p \alpha+2}-\frac{b^{p}}{p \alpha+2}
$$$$
\left.-b^{p} \int_{0}^{1 / 2} t^{p \alpha}(1-t) d t\right)^{1 / p}
$$$$
\leq \frac{\ln b-\ln a}{2}\left(\frac{\left|f^{\prime}(a)\right|^{q}+2\left|f^{\prime}(b)\right|^{q}-\left|f^{\prime}(b)\right|^{q}}{2}\right)^{1 / q}
$$$$
\times\left(\frac{a^{p}-2 b^{p}}{p \alpha+2}+\frac{b^{p}}{p \alpha+1}-\frac{2 b^{p}+a^{p}}{2^{p \alpha+2}(p \alpha+1)}\right.
$$ 


$$
\begin{aligned}
& +\frac{2 b^{p}-a^{p}}{2^{p \alpha+2}(p \alpha+2)}-\frac{a^{p}}{p \alpha(p \alpha+1)} \\
& -\frac{a^{p}+b^{p}}{2^{p \alpha+2}(p \alpha+1)(p \alpha+2)} \\
& \left.+\frac{2 a^{p}}{(p \alpha+1)(p \alpha+2)}\right)^{1 / p} .
\end{aligned}
$$

The proof is done.

Theorem 12. Let $f:[0, b] \rightarrow \mathbb{R}^{+}$be a differentiable mapping. If $\left|f^{\prime}\right|$ is measurable and $\left|f^{\prime}\right|$ is GG-convex on $[a, b]$ for some fired $\alpha \in(0, \infty), t \in[0,1]$ and $k>0,0 \leq a<b$, then the following integrals hold:

$$
\begin{aligned}
\mid \frac{f(a)+f(b)}{2} & -\frac{\Gamma(\alpha+1)}{2(\ln b-\ln a)^{\alpha}}\left[{ }_{H} J_{a^{+}}^{\alpha} f(b)+{ }_{H} J_{b^{-}}^{\alpha} f(a)\right] \mid \\
\leq & b\left|f^{\prime}(b)\right| \frac{\ln b-\ln a}{2} \\
& \times\left\{\frac{a\left|f^{\prime}(a)\right|}{b\left|f^{\prime}(b)\right|}\right. \\
& \times \sum_{i=1}^{\infty}(-1)^{i-1} \frac{\left[\ln \left(a\left|f^{\prime}(a)\right| / b\left|f^{\prime}(b)\right|\right)\right]^{i-1}}{(\alpha+1)_{i}} \\
& +\sum_{i=1}^{\infty} \frac{\left[\ln \left(a\left|f^{\prime}(a)\right| / b\left|f^{\prime}(b)\right|\right)\right]^{i-1}}{(\alpha+1)_{i}} \\
& -\frac{(1 / 2)^{\alpha-1}\left(a\left|f^{\prime}(a)\right| / b\left|f^{\prime}(b)\right|\right)^{1 / 2}}{\alpha+1} \\
& +2 \frac{1-(1 / 2)^{\alpha}\left(a\left|f^{\prime}(a)\right| / b\left|f^{\prime}(b)\right|\right)^{1 / 2}}{\ln \left(a\left|f^{\prime}(a)\right| / b\left|f^{\prime}(b)\right|\right)}
\end{aligned}
$$

Proof. By using Definition 4 and Lemmas 1, 7, 8, and 9, we have

$$
\begin{gathered}
\left|\frac{f(a)+f(b)}{2}-\frac{\Gamma(\alpha+1)}{2(\ln b-\ln a)^{\alpha}}\left[{ }_{H} J_{a^{+}}^{\alpha} f(b)+{ }_{H} J_{b^{-}}^{\alpha} f(a)\right]\right| \\
\leq \frac{\ln b-\ln a}{2} \int_{1 / 2}^{1} a^{t} b^{1-t}\left[t^{\alpha}-(1-t)^{\alpha}\right]\left|f^{\prime}\left(a^{t} b^{1-t}\right)\right| d t \\
+\frac{\ln b-\ln a}{2} \int_{0}^{1 / 2} a^{t} b^{1-t}\left[(1-t)^{\alpha}-t^{\alpha}\right] \\
\times\left|f^{\prime}\left(a^{t} b^{1-t}\right)\right| d t
\end{gathered}
$$

$$
\begin{aligned}
& \leq \frac{\ln b-\ln a}{2} \int_{1 / 2}^{1} a^{t} b^{1-t}\left[t^{\alpha}-(1-t)^{\alpha}\right] \\
& \times\left|f^{\prime}(a)\right|^{t}\left|f^{\prime}(b)\right|^{1-t} d t \\
& +\frac{\ln b-\ln a}{2} \int_{0}^{1 / 2} a^{t} b^{1-t}\left[(1-t)^{\alpha}-t^{\alpha}\right] \\
& \times\left|f^{\prime}(a)\right|^{t}\left|f^{\prime}(b)\right|^{1-t} d t \\
& \leq b\left|f^{\prime}(b)\right| \frac{\ln b-\ln a}{2} \\
& \times \int_{1 / 2}^{1}\left(\frac{a\left|f^{\prime}(a)\right|}{b\left|f^{\prime}(b)\right|}\right)^{t}\left[t^{\alpha}-(1-t)^{\alpha}\right] d t \\
& +b\left|f^{\prime}(b)\right| \frac{\ln b-\ln a}{2} \\
& \times \int_{0}^{1 / 2}\left(\frac{a\left|f^{\prime}(a)\right|}{b\left|f^{\prime}(b)\right|}\right)^{t}\left[(1-t)^{\alpha}-t^{\alpha}\right] d t \\
& \leq b\left|f^{\prime}(b)\right| \frac{\ln b-\ln a}{2} \\
& \times\left\{\int_{0}^{1}\left(\frac{a\left|f^{\prime}(a)\right|}{b\left|f^{\prime}(b)\right|}\right)^{t} t^{\alpha} d t\right. \\
& -2 \int_{0}^{1 / 2}\left(\frac{a\left|f^{\prime}(a)\right|}{b\left|f^{\prime}(b)\right|}\right)^{t} t^{\alpha} d t \\
& -\int_{0}^{1}\left(\frac{a\left|f^{\prime}(a)\right|}{b\left|f^{\prime}(b)\right|}\right)^{t}(1-t)^{\alpha} d t \\
& \left.+2 \int_{0}^{1 / 2}\left(\frac{a\left|f^{\prime}(a)\right|}{b\left|f^{\prime}(b)\right|}\right)^{t}(1-t)^{\alpha} d t\right\} \\
& \leq b\left|f^{\prime}(b)\right| \frac{\ln b-\ln a}{2} \\
& \times\left\{\frac{a\left|f^{\prime}(a)\right|}{b\left|f^{\prime}(b)\right|}\right.
\end{aligned}
$$$$
\times \sum_{i=1}^{\infty}(-1)^{i-1} \frac{\left[\ln \left(a\left|f^{\prime}(a)\right| / b\left|f^{\prime}(b)\right|\right)\right]^{i-1}}{(\alpha+1)_{i}}
$$$$
-\sum_{i=1}^{\infty} \frac{\left[\ln \left(a\left|f^{\prime}(a)\right| / b\left|f^{\prime}(b)\right|\right)\right]^{i-1}}{(\alpha+1)_{i}}
$$$$
-\left(\frac{1}{2}\right)^{\alpha}\left(\frac{a\left|f^{\prime}(a)\right|}{b\left|f^{\prime}(b)\right|}\right)^{1 / 2}
$$$$
\times \sum_{i=1}^{\infty} \frac{\left[-(1 / 2) \ln \left(a\left|f^{\prime}(a)\right| / b\left|f^{\prime}(b)\right|\right)\right]^{i-1}}{(\alpha+1)_{i}}
$$ 


$$
\begin{aligned}
& +2 \sum_{i=0}^{\infty} \frac{\left[\ln \left(a\left|f^{\prime}(a)\right| / b\left|f^{\prime}(b)\right|\right)\right]^{i-1}}{(\alpha+1)_{i}} \\
& \left.\times\left[1-\left(\frac{1}{2}\right)^{\alpha+i}\left(\frac{a\left|f^{\prime}(a)\right|}{b\left|f^{\prime}(b)\right|}\right)^{1 / 2}\right]\right\} \\
& \leq b\left|f^{\prime}(b)\right| \frac{\ln b-\ln a}{2} \\
& \times\left\{\frac{a\left|f^{\prime}(a)\right|}{b\left|f^{\prime}(b)\right|}\right. \\
& \times \sum_{i=1}^{\infty}(-1)^{i-1} \frac{\left[\ln \left(a\left|f^{\prime}(a)\right| / b\left|f^{\prime}(b)\right|\right)\right]^{i-1}}{(\alpha+1)_{i}} \\
& -\sum_{i=1}^{\infty} \frac{\left[\ln \left(a\left|f^{\prime}(a)\right| / b\left|f^{\prime}(b)\right|\right)\right]^{i-1}}{(\alpha+1)_{i}} \\
& -\left(\frac{1}{2}\right)^{\alpha}\left(\frac{a\left|f^{\prime}(a)\right|}{b\left|f^{\prime}(b)\right|}\right)^{1 / 2} \\
& \times \sum_{i=1}^{\infty} \frac{\left[-(1 / 2) \ln \left(a\left|f^{\prime}(a)\right| / b\left|f^{\prime}(b)\right|\right)\right]^{i-1}}{(\alpha+1)_{i}} \\
& +2 \frac{1-(1 / 2)^{\alpha}\left(a\left|f^{\prime}(a)\right| / b\left|f^{\prime}(b)\right|\right)^{1 / 2}}{\ln \left(a\left|f^{\prime}(a)\right| / b\left|f^{\prime}(b)\right|\right)} \\
& +2 \sum_{i=1}^{\infty} \frac{\left[\ln \left(a\left|f^{\prime}(a)\right| / b\left|f^{\prime}(b)\right|\right)\right]^{i-1}}{(\alpha+1)_{i}} \\
& -\left(\frac{1}{2}\right)^{\alpha}\left(\frac{a\left|f^{\prime}(a)\right|}{b\left|f^{\prime}(b)\right|}\right)^{1 / 2} \\
& \left.\times \sum_{i=1}^{\infty} \frac{\left[(1 / 2) \ln \left(a\left|f^{\prime}(a)\right| / b\left|f^{\prime}(b)\right|\right)\right]^{i-1}}{(\alpha+1)_{i}}\right\} \\
& \leq b\left|f^{\prime}(b)\right| \frac{\ln b-\ln a}{2} \\
& \times\left\{\frac{a\left|f^{\prime}(a)\right|}{b\left|f^{\prime}(b)\right|}\right. \\
& \times \sum_{i=1}^{\infty}(-1)^{i-1} \frac{\left[\ln \left(a\left|f^{\prime}(a)\right| / b\left|f^{\prime}(b)\right|\right)\right]^{i-1}}{(\alpha+1)_{i}} \\
& +\sum_{i=1}^{\infty} \frac{\left[\ln \left(a\left|f^{\prime}(a)\right| / b\left|f^{\prime}(b)\right|\right)\right]^{i-1}}{(\alpha+1)_{i}}
\end{aligned}
$$

$$
\begin{aligned}
& -\frac{(1 / 2)^{\alpha-1}\left(a\left|f^{\prime}(a)\right| / b\left|f^{\prime}(b)\right|\right)^{1 / 2}}{\alpha+1} \\
& \left.+2 \frac{1-(1 / 2)^{\alpha}\left(a\left|f^{\prime}(a)\right| / b\left|f^{\prime}(b)\right|\right)^{1 / 2}}{\ln \left(a\left|f^{\prime}(a)\right| / b\left|f^{\prime}(b)\right|\right)}\right\} .
\end{aligned}
$$

The proof is done.

Theorem 13. Let $f:[0, b] \rightarrow \mathbb{R}^{+}$be a differentiable mapping. If $\left|f^{\prime}\right|^{q}$ is measurable and $\left|f^{\prime}\right|^{q},(q>1)$ is GG-convex on $[a, b]$, for some fired $\alpha \in(0, \infty), t \in[0,1]$, and $k>0,0 \leq a<b$, then the following integrals hold:

$$
\begin{gathered}
\left|\frac{f(a)+f(b)}{2}-\frac{\Gamma(\alpha+1)}{2(\ln b-\ln a)^{\alpha}}\left[{ }_{H} J_{a^{+}}^{\alpha} f(b)+{ }_{H} J_{b^{-}}^{\alpha} f(a)\right]\right| \\
\leq \frac{\ln b-\ln a}{2} \\
\quad \times\left(a^{p} \sum_{i=1}^{\infty}(-1)^{i-1} \frac{\left[(1 / 2) \ln (a / b)^{p}\right]^{i-1}}{(p \alpha+1)_{i}}\right. \\
\quad+b^{p} \sum_{i=1}^{\infty} \frac{\left[\ln (a / b)^{p}\right]^{i-1}}{(p \alpha+1)_{i}}-\frac{(1 / 2)^{p \alpha-1}\left(a b^{p / 2}\right)}{p \alpha+1} \\
\left.\quad-\left[\ln \left(\frac{a}{b}\right)^{p}\right]^{-1}\left[\left(\frac{1}{2}\right)^{p \alpha-1}(a b)^{p / 2}+2 b^{p}\right]\right)^{1 / p} \\
\quad \times\left(\frac{\left|f^{\prime}(b)\right|^{q}}{\ln \left(\left|f^{\prime}(a)\right| /\left|f^{\prime}(b)\right|\right)^{q}}\left[\left|\frac{f^{\prime}(a)}{f^{\prime}(b)}\right|^{q}-1\right]\right)^{1 / q}
\end{gathered}
$$

where $1 / p+1 / q=1$.

Proof. By using Definition 4 and Lemmas 1, 7, 8, and 9, we have

$$
\begin{gathered}
\left|\frac{f(a)+f(b)}{2}-\frac{\Gamma(\alpha+1)}{2(\ln b-\ln a)^{\alpha}}\left[{ }_{H} J_{a^{+}}^{\alpha} f(b)+{ }_{H} J_{b^{-}}^{\alpha} f(a)\right]\right| \\
\leq \frac{\ln b-\ln a}{2}\left(\int_{0}^{1}\left(a^{t} b^{1-t}\left|(1-t)^{\alpha}-t^{\alpha}\right|\right)^{p} d t\right)^{1 / p} \\
\times\left(\int_{0}^{1}\left|f^{\prime}\left(a^{t} b^{1-t}\right)\right|^{q} d t\right)^{1 / q} \\
\leq \frac{\ln b-\ln a}{2}\left(b^{p} \int_{1 / 2}^{1}\left(\frac{a}{b}\right)^{p t} t^{p \alpha} d t\right. \\
-b^{p} \int_{1 / 2}^{1}\left(\frac{a}{b}\right)^{p t}(1-t)^{p \alpha} d t \\
+b^{p} \int_{0}^{1 / 2}\left(\frac{a}{b}\right)^{p t}(1-t)^{p \alpha} d t \\
\left.-b^{p} \int_{0}^{1 / 2}\left(\frac{a}{b}\right)^{p t} t^{p \alpha} d t\right)^{1 / p}
\end{gathered}
$$




$$
\begin{aligned}
& \times\left(\frac{\left|f^{\prime}(b)\right|^{q}}{\ln \left(\left|f^{\prime}(a)\right| /\left|f^{\prime}(b)\right|\right)^{q}}\right. \\
& \left.\times \int_{0}^{1} \ln \left(\frac{\left|f^{\prime}(a)\right|}{\left|f^{\prime}(b)\right|}\right)^{q}\left[\frac{\left|f^{\prime}(a)\right|}{\left|f^{\prime}(b)\right|}\right]^{q t} d t\right)^{1 / q} \\
& \leq \frac{\ln b-\ln a}{2}\left(b^{p}\left(\frac{a}{b}\right)^{p} \sum_{i=1}^{\infty}(-1)^{i-1} \frac{\left[\ln (a / b)^{p}\right]^{i-1}}{(p \alpha+1)_{i}}\right. \\
& -b^{p} \sum_{i=0}^{\infty} \frac{\left[\ln (a / b)^{p}\right]^{i-1}}{(p \alpha+1)_{i}} \\
& -2 b^{p} \int_{0}^{1 / 2}\left(\frac{a}{b}\right)^{p t} t^{p \alpha} d t \\
& \left.+2 b^{p} \int_{0}^{1 / 2}\left(\frac{a}{b}\right)^{p t}(1-t)^{p \alpha} d t\right)^{1 / p} \\
& \times\left(\frac{\left|f^{\prime}(b)\right|^{q}}{\ln \left(\left|f^{\prime}(a)\right| /\left|f^{\prime}(b)\right|\right)^{q}}\left[\left|\frac{f^{\prime}(a)}{f^{\prime}(b)}\right|^{q}-1\right]\right)^{1 / q} \\
& \leq \frac{\ln b-\ln a}{2} \\
& \times\left(b^{p}\left(\frac{a}{b}\right)^{p} \sum_{i=1}^{\infty}(-1)^{i-1} \frac{\left[\ln (a / b)^{p}\right]^{i-1}}{(p \alpha+1)_{i}}\right. \\
& -b^{p} \sum_{i=1}^{\infty} \frac{\left[\ln (a / b)^{p}\right]^{i-1}}{(p \alpha+1)_{i}} \\
& -\left(\frac{1}{2}\right)^{p \alpha} b^{p}\left(\frac{a}{b}\right)^{p / 2} \sum_{i=1}^{\infty} \frac{\left[-(1 / 2) \ln (a / b)^{p}\right]^{i-1}}{(p \alpha+1)_{i}} \\
& +2 b^{p} \sum_{i=0}^{\infty} \frac{\left[\ln (a / b)^{p}\right]^{i-1}}{(p \alpha+1)_{i}} \\
& \left.\times\left[1-\left(\frac{a}{b}\right)^{p / 2}\left(1-\frac{1}{2}\right)^{p \alpha+i}\right]\right)^{1 / p} \\
& \times\left(\frac{\left|f^{\prime}(b)\right|^{q}}{\ln \left(\left|f^{\prime}(a)\right| /\left|f^{\prime}(b)\right|\right)^{q}}\left[\left|\frac{f^{\prime}(a)}{f^{\prime}(b)}\right|^{q}-1\right]\right)^{1 / q} \\
& \leq \frac{\ln b-\ln a}{2} \\
& \times\left(a^{p} \sum_{i=1}^{\infty}(-1)^{i-1} \frac{\left[\ln (a / b)^{p}\right]^{i-1}}{(p \alpha+1)_{i}}\right. \\
& -b^{p} \sum_{i=1}^{\infty} \frac{\left[\ln (a / b)^{p}\right]^{i-1}}{(p \alpha+1)_{i}} \\
& -\left(\frac{1}{2}\right)^{p \alpha}(a b)^{p / 2} \sum_{i=1}^{\infty} \frac{\left[-(1 / 2) \ln (a / b)^{p}\right]^{i-1}}{(p \alpha+1)_{i}}
\end{aligned}
$$

$$
\begin{aligned}
& +2 b^{p} \sum_{i=0}^{\infty} \frac{\left[\ln (a / b)^{p}\right]^{i-1}}{(p \alpha+1)_{i}} \\
& \left.-2 b^{p}\left(\frac{a}{b}\right)^{p / 2} \sum_{i=0}^{\infty}\left(1-\frac{1}{2}\right)^{p \alpha+i} \frac{\left[\ln (a / b)^{p}\right]^{i-1}}{(p \alpha+1)_{i}}\right)^{1 / p} \\
& \times\left(\frac{\left|f^{\prime}(b)\right|^{q}}{\ln \left(\left|f^{\prime}(a)\right| /\left|f^{\prime}(b)\right|\right)^{q}}\left[\left|\frac{f^{\prime}(a)}{f^{\prime}(b)}\right|^{q}-1\right]\right)^{1 / q} \\
& \leq \frac{\ln b-\ln a}{2} \\
& \times\left(a^{p} \sum_{i=1}^{\infty}(-1)^{i-1} \frac{\left[\ln (a / b)^{p}\right]^{i-1}}{(p \alpha+1)_{i}}-b^{p} \sum_{i=1}^{\infty} \frac{\left[\ln (a / b)^{p}\right]^{i-1}}{(p \alpha+1)_{i}}\right. \\
& -\left(\frac{1}{2}\right)^{p \alpha}(a b)^{p / 2} \sum_{i=1}^{\infty} \frac{\left[-(1 / 2) \ln (a / b)^{p}\right]^{i-1}}{(p \alpha+1)_{i}} \\
& +2 b^{p} \sum_{i=1}^{\infty} \frac{\left[\ln (a / b)^{p}\right]^{i-1}}{(p \alpha+1)_{i}}+2 b^{p}\left[\ln (a / b)^{p}\right]^{-1} \\
& \left.-2 b^{p}\left(\frac{a}{b}\right)^{p / 2} \sum_{i=0}^{\infty}\left(1-\frac{1}{2}\right)^{p \alpha+i} \frac{\left[\ln (a / b)^{p}\right]^{i-1}}{(p \alpha+1)_{i}}\right)^{1 / p} \\
& \times\left(\frac{\left|f^{\prime}(b)\right|^{q}}{\ln \left(\left|f^{\prime}(a)\right| /\left|f^{\prime}(b)\right|\right)^{q}}\left[\left|\frac{f^{\prime}(a)}{f^{\prime}(b)}\right|^{q}-1\right]\right)^{1 / q} \\
& \leq \frac{\ln b-\ln a}{2} \\
& \times\left(a^{p} \sum_{i=1}^{\infty}(-1)^{i-1} \frac{\left[\ln (a / b)^{p}\right]^{i-1}}{(p \alpha+1)_{i}}+b^{p} \sum_{i=1}^{\infty} \frac{\left[\ln (a / b)^{p}\right]^{i-1}}{(p \alpha+1)_{i}}\right. \\
& -\left(\frac{1}{2}\right)^{p \alpha}(a b)^{p / 2} \sum_{i=1}^{\infty} \frac{\left[-(1 / 2) \ln (a / b)^{p}\right]^{i-1}}{(p \alpha+1)_{i}} \\
& -\left(\frac{1}{2}\right)^{p \alpha}(a b)^{p / 2} \sum_{i=0}^{\infty}\left(\frac{1}{2}\right)^{i-1} \frac{\left[\ln (a / b)^{p}\right]^{i-1}}{(p \alpha+1)_{i}} \\
& \left.+2 b^{p}\left[\ln \left(\frac{a}{b}\right)^{p}\right]^{-1}\right)^{1 / p} \\
& \times\left(\frac{\left|f^{\prime}(b)\right|^{q}}{\ln \left(\left|f^{\prime}(a)\right| /\left|f^{\prime}(b)\right|\right)^{q}}\left[\left|\frac{f^{\prime}(a)}{f^{\prime}(b)}\right|^{q}-1\right]\right)^{1 / q} \\
& \leq \frac{\ln b-\ln a}{2} \\
& \times\left(a^{p} \sum_{i=1}^{\infty}(-1)^{i-1} \frac{\left[\ln (a / b)^{p}\right]^{i-1}}{(p \alpha+1)_{i}}+b^{p} \sum_{i=1}^{\infty} \frac{\left[\ln (a / b)^{p}\right]^{i-1}}{(p \alpha+1)_{i}}\right. \\
& -\left(\frac{1}{2}\right)^{p \alpha}(a b)^{p / 2} \sum_{i=1}^{\infty} \frac{\left[-(1 / 2) \ln (a / b)^{p}\right]^{i-1}}{(p \alpha+1)_{i}}
\end{aligned}
$$




$$
\begin{aligned}
& -\left(\frac{1}{2}\right)^{p \alpha}(a b)^{p / 2} \sum_{i=1}^{\infty} \frac{\left[(1 / 2) \ln (a / b)^{p}\right]^{i-1}}{(p \alpha+1)_{i}} \\
& -\left(\frac{1}{2}\right)^{p \alpha-1}(a b)^{p / 2}\left[\ln \left(\frac{a}{b}\right)^{p}\right]^{-1} \\
& \left.+2 b^{p}\left[\ln \left(\frac{a}{b}\right)^{p}\right]^{-1}\right)^{1 / p} \\
& \times\left(\frac{\left|f^{\prime}(b)\right|^{q}}{\ln \left(\left|f^{\prime}(a)\right| /\left|f^{\prime}(b)\right|\right)^{q}}\left[\left|\frac{f^{\prime}(a)}{f^{\prime}(b)}\right|^{q}-1\right]\right)^{1 / q} \\
& \leq \frac{\ln b-\ln a}{2} \\
& \times\left(a^{p} \sum_{i=1}^{\infty}(-1)^{i-1} \frac{\left[\ln (a / b)^{p}\right]^{i-1}}{(p \alpha+1)_{i}}+b^{p} \sum_{i=1}^{\infty} \frac{\left[\ln (a / b)^{p}\right]^{i-1}}{(p \alpha+1)_{i}}\right. \\
& -2\left(\frac{1}{2}\right)^{p \alpha}(a b)^{p / 2} \frac{1}{p \alpha+1} \\
& \left.-\left[\ln \left(\frac{a}{b}\right)^{p}\right]^{-1}\left[\left(\frac{1}{2}\right)^{p \alpha-1}(a b)^{p / 2}+2 b^{p}\right]\right)^{1 / p} \\
& \times\left(\frac{\left|f^{\prime}(b)\right|^{q}}{\ln \left(\left|f^{\prime}(a)\right| /\left|f^{\prime}(b)\right|\right)^{q}}\left[\left|\frac{f^{\prime}(a)}{f^{\prime}(b)}\right|^{q}-1\right]\right)^{1 / q} \\
& \leq \frac{\ln b-\ln a}{2} \\
& \times\left(a^{p} \sum_{i=1}^{\infty}(-1)^{i-1} \frac{\left[(1 / 2) \ln (a / b)^{p}\right]^{i-1}}{(p \alpha+1)_{i}}\right. \\
& +b^{p} \sum_{i=1}^{\infty} \frac{\left[\ln (a / b)^{p}\right]^{i-1}}{(p \alpha+1)_{i}}-\frac{(1 / 2)^{p \alpha-1}\left(a b^{p / 2}\right)}{p \alpha+1} \\
& \left.-\left[\ln \left(\frac{a}{b}\right)^{p}\right]^{-1}\left[\left(\frac{1}{2}\right)^{p \alpha-1}(a b)^{p / 2}+2 b^{p}\right]\right)^{1 / p} \\
& \times\left(\frac{\left|f^{\prime}(b)\right|^{q}}{\ln \left(\left|f^{\prime}(a)\right| /\left|f^{\prime}(b)\right|\right)^{q}}\left[\left|\frac{f^{\prime}(a)}{f^{\prime}(b)}\right|^{q}-1\right]\right)^{1 / q} .
\end{aligned}
$$

The proof is done.

\section{Main Results Based on Lemma 2}

Theorem 14. Let $f:[0, b] \rightarrow \mathbb{R}^{+}$be a differentiable mapping. If $\left|f^{\prime}\right|$ is measurable and $\left|f^{\prime}\right|$ is GG-convex on $[a, b]$, for some fired $\alpha \in(0, \infty)$ and $t \in[0,1], 0 \leq a<b$, then the following integrals hold:

$$
\begin{aligned}
&\left|\frac{\Gamma(\alpha+1)}{2(\ln b-\ln a)^{\alpha}}\left[{ }_{H} J_{a^{+}}^{\alpha} f(b)+{ }_{H} J_{b^{-}}^{\alpha} f(a)\right]-f\left(\frac{a+b}{2}\right)\right| \\
& \leq \frac{\left|f^{\prime}(b)\right|-\left|f^{\prime}(a)\right|}{2}+\frac{\ln b-\ln a}{2} \\
& \times\left\{\left(1-\frac{1}{2^{\alpha+1}}\right)\right. \\
& \times\left[\frac{b\left|f^{\prime}(b)\right|}{\alpha+1}+\frac{b\left|f^{\prime}(a)\right|+a\left|f^{\prime}(b)\right|-2 b\left|f^{\prime}(b)\right|}{\alpha+2}\right] \\
&+\frac{a\left|f^{\prime}(a)\right|-a\left|f^{\prime}(b)\right|-b\left|f^{\prime}(a)\right|}{\alpha+3} \\
&-\frac{a\left|f^{\prime}(a)\right|-b\left|f^{\prime}(a)\right|}{(\alpha+3) 2^{\alpha+3}}+\frac{2 a\left|f^{\prime}(a)\right|}{(\alpha+1)(\alpha+2)(\alpha+3)} \\
&+\frac{b\left|f^{\prime}(a)\right|+a\left|f^{\prime}(b)\right|-2 a\left|f^{\prime}(a)\right|}{(\alpha+1)(\alpha+3)} \\
&+\frac{2 a\left(\left|f^{\prime}(a)\right|-\left|f^{\prime}(b)\right|\right)-2 b\left|f^{\prime}(a)\right|}{2^{\alpha+3}(\alpha+2)(\alpha+3)} \\
&+\frac{b\left|f^{\prime}(a)\right|+a\left|f^{\prime}(b)\right|}{2^{\alpha+2}(\alpha+2)}+\frac{a\left|f^{\prime}(a)\right|}{(\alpha+1) 2^{\alpha+2}} \\
& \frac{a\left|f^{\prime}(a)\right|}{\left.(\alpha+2)^{2} 2^{\alpha+2}\right\}} .
\end{aligned}
$$

Proof. By using Definition 4 and Lemmas 2 and 6, we have

$$
\begin{aligned}
& \left|\frac{\Gamma(\alpha+1)}{2(\ln b-\ln a)^{\alpha}}\left[{ }_{H} J_{a^{+}}^{\alpha} f(b)+{ }_{H} J_{b^{-}}^{\alpha} f(a)\right]-f\left(\frac{a+b}{2}\right)\right| \\
& \leq \frac{b-a}{2} \int_{0}^{1}\left|k f^{\prime}(t a+(1-t) b)\right| d t \\
& \quad+\frac{\ln b-\ln a}{2} \int_{0}^{1} a^{t} b^{1-t}\left|(1-t)^{\alpha}-t^{\alpha}\right| \\
& \quad \times\left|f^{\prime}\left(a^{t} b^{1-t}\right)\right| d t \\
& \leq \frac{-1}{2}(|f(a)|-|f(b)|) \\
& \quad+\frac{\ln b-\ln a}{2} \\
& \quad \times\left\{\int_{1 / 2}^{1} a^{t} b^{1-t}\left[t^{\alpha}-(1-t)^{\alpha}\right]\left|f^{\prime}\left(a^{t} b^{1-t}\right)\right| d t\right. \\
& \left.\quad+\int_{0}^{1 / 2} a^{t} b^{1-t}\left[(1-t)^{\alpha}-t^{\alpha}\right]\left|f^{\prime}\left(a^{t} b^{1-t}\right)\right| d t\right\}
\end{aligned}
$$




$$
\begin{aligned}
& \leq \frac{-1}{2}(|f(a)|-|f(b)|) \\
& \leq \frac{\left|f^{\prime}(b)\right|-\left|f^{\prime}(a)\right|}{2} \\
& +\frac{\ln b-\ln a}{2} \\
& \times\left\{\int_{1 / 2}^{1} t^{\alpha}[a t+b(1-t)]\right. \\
& \times\left[t\left|f^{\prime}(a)\right|+(1-t)\left|f^{\prime}(b)\right|\right] d t \\
& -\int_{1 / 2}^{1}(1-t)^{\alpha}[a t+b(1-t)] \\
& \left.\times\left[t\left|f^{\prime}(a)\right|+(1-t)\left|f^{\prime}(b)\right|\right] d t\right\} \\
& +\frac{\ln b-\ln a}{2} \\
& \times\left\{\int_{0}^{1 / 2}(1-t)^{\alpha}[a t+b(1-t)]\right. \\
& \times\left[t\left|f^{\prime}(a)\right|+(1-t)\left|f^{\prime}(b)\right|\right] d t \\
& -\int_{0}^{1 / 2} t^{\alpha}[a t+b(1-t)] \\
& \left.\times\left[t\left|f^{\prime}(a)\right|+(1-t)\left|f^{\prime}(b)\right|\right] d t\right\} \\
& \leq \frac{-1}{2}(|f(a)|-|f(b)|) \\
& +\frac{\ln b-\ln a}{2} \\
& \times\left\{\left|f^{\prime}(a)\right| \int_{1 / 2}^{1} t^{\alpha+1}[a t+b(1-t)] d t\right. \\
& +\left|f^{\prime}(b)\right| \int_{1 / 2}^{1}(1-t) t^{\alpha}[a t+b(1-t)] d t \\
& -\left|f^{\prime}(a)\right| \int_{1 / 2}^{1}(1-t)^{\alpha} t[a t+b(1-t)] d t \\
& \left.-\left|f^{\prime}(b)\right| \int_{1 / 2}^{1}(1-t)^{\alpha+1}[a t+b(1-t)] d t\right\} \\
& +\frac{\ln b-\ln a}{2} \\
& \times\left\{\left|f^{\prime}(a)\right| \int_{0}^{1 / 2} t(1-t)^{\alpha}[a t+b(1-t)] d t\right. \\
& +\left|f^{\prime}(b)\right| \int_{0}^{1 / 2}(1-t)^{\alpha+1}[a t+b(1-t)] d t \\
& -\left|f^{\prime}(a)\right| \int_{0}^{1 / 2} t^{\alpha+1}[a t+b(1-t)] d t \\
& \left.-\left|f^{\prime}(b)\right| \int_{0}^{1 / 2}(1-t) t^{\alpha}[a t+b(1-t)] d t\right\} \\
& +\frac{\ln b-\ln a}{2} \\
& \times\left\{\frac{a\left|f^{\prime}(a)\right|}{\alpha+3}\left(1-\frac{1}{2^{\alpha+3}}\right)+\frac{b\left|f^{\prime}(a)\right|}{\alpha+2}\left(1-\frac{1}{2^{\alpha+2}}\right)\right. \\
& -\frac{b\left|f^{\prime}(a)\right|}{\alpha+3}\left(1-\frac{1}{2^{\alpha+3}}\right)+\frac{a\left|f^{\prime}(b)\right|}{\alpha+2}\left(1-\frac{1}{2^{\alpha+2}}\right) \\
& -\frac{a\left|f^{\prime}(b)\right|}{\alpha+3}\left(1-\frac{1}{2^{\alpha+3}}\right)+\frac{b\left|f^{\prime}(b)\right|}{\alpha+1}\left(1-\frac{1}{2^{\alpha+1}}\right) \\
& -\frac{2 b\left|f^{\prime}(b)\right|}{\alpha+2}\left(1-\frac{1}{2^{\alpha+2}}\right)+\frac{b\left|f^{\prime}(b)\right|}{\alpha+3}\left(1-\frac{1}{2^{\alpha+3}}\right) \\
& -a\left|f^{\prime}(a)\right| \int_{1 / 2}^{1}(1-t)^{\alpha} t^{2} d t \\
& -b\left|f^{\prime}(a)\right| \int_{1 / 2}^{1} t(1-t)^{\alpha+1} d t \\
& -a\left|f^{\prime}(b)\right| \int_{1 / 2}^{1} t(1-t)^{\alpha+1} d t \\
& \left.-\frac{b\left|f^{\prime}(b)\right|}{\alpha+3} \int_{1 / 2}^{1}(\alpha+3)(1-t)^{\alpha+2} d t\right\} \\
& +\frac{\ln b-\ln a}{2} \\
& \times\left\{a\left|f^{\prime}(a)\right| \int_{0}^{1 / 2} t^{2}(1-t)^{\alpha} d t\right. \\
& +b\left|f^{\prime}(a)\right| \int_{0}^{1 / 2} t(1-t)^{\alpha+1} d t \\
& +a\left|f^{\prime}(b)\right| \int_{0}^{1 / 2} t(1-t)^{\alpha+1} d t \\
& +\frac{b\left|f^{\prime}(b)\right|}{\alpha+3}\left(\frac{1}{2^{\alpha+3}}-1\right)-\frac{a\left|f^{\prime}(a)\right|}{\alpha+3} \frac{1}{2^{\alpha+3}} \\
& -\frac{b\left|f^{\prime}(a)\right|}{\alpha+2} \frac{1}{2^{\alpha+2}}+\frac{b\left|f^{\prime}(a)\right|}{\alpha+3} \frac{1}{2^{\alpha+3}} \\
& -\frac{a\left|f^{\prime}(b)\right|}{\alpha+2} \frac{1}{2^{\alpha+2}}+\frac{a\left|f^{\prime}(b)\right|}{\alpha+3} \frac{1}{2^{\alpha+3}}-\frac{b\left|f^{\prime}(b)\right|}{\alpha+1} \\
& \left.\times \frac{1}{2^{\alpha+1}}+\frac{2 b\left|f^{\prime}(b)\right|}{\alpha+2} \frac{1}{2^{\alpha+2}}-\frac{b\left|f^{\prime}(a)\right|}{\alpha+3} \frac{1}{2^{\alpha+3}}\right\}
\end{aligned}
$$




$$
\begin{aligned}
& \leq \frac{\left|f^{\prime}(b)\right|-\left|f^{\prime}(a)\right|}{2} \\
& \leq \frac{\left|f^{\prime}(b)\right|-\left|f^{\prime}(a)\right|}{2} \\
& +\frac{\ln b-\ln a}{2} \\
& +\frac{\ln b-\ln a}{2} \\
& \times\left\{\frac{(a-b)\left[\left|f^{\prime}(a)\right|-\left|f^{\prime}(b)\right|\right]}{\alpha+3}\left(1-\frac{1}{2^{\alpha+3}}\right)\right. \\
& \times\left\{\frac{(a-b)\left[\left|f^{\prime}(a)\right|-\left|f^{\prime}(b)\right|\right]}{\alpha+3}\left(1-\frac{1}{2^{\alpha+3}}\right)\right. \\
& +\frac{b\left|f^{\prime}(b)\right|}{\alpha+1}\left(1-\frac{1}{2^{\alpha+1}}\right) \\
& +\frac{b\left|f^{\prime}(b)\right|}{\alpha+1}\left(1-\frac{1}{2^{\alpha+1}}\right) \\
& +\frac{b\left[\left|f^{\prime}(a)\right|-\left|f^{\prime}(b)\right|\right]+(a-b)\left|f^{\prime}(b)\right|}{\alpha+2} \\
& \times\left(1-\frac{1}{2^{\alpha+2}}\right)-a\left|f^{\prime}(a)\right| \int_{0}^{1}(1-t)^{\alpha} t^{2} d t \\
& +a\left|f^{\prime}(a)\right| \int_{0}^{1 / 2}(1-t)^{\alpha} t^{2} d t \\
& -b\left|f^{\prime}(a)\right| \int_{0}^{1} t(1-t)^{\alpha+1} d t \\
& +b\left|f^{\prime}(a)\right| \int_{0}^{1 / 2} t(1-t)^{\alpha+1} d t \\
& -a\left|f^{\prime}(b)\right| \int_{0}^{1} t(1-t)^{\alpha+1} d t \\
& \left.+a\left|f^{\prime}(b)\right| \int_{0}^{1 / 2} t(1-t)^{\alpha+1}\right\} \\
& +\frac{\ln b-\ln a}{2} \\
& \times\left\{a\left|f^{\prime}(a)\right| \int_{0}^{1 / 2} t^{2}(1-t)^{\alpha} d t\right. \\
& +b\left|f^{\prime}(a)\right| \int_{0}^{1 / 2} t(1-t)^{\alpha+1} d t \\
& +a\left|f^{\prime}(b)\right| \int_{0}^{1 / 2} t(1-t)^{\alpha+1} d t \\
& +\frac{b\left|f^{\prime}(b)\right|}{\alpha+3}\left(\frac{1}{2^{\alpha+3}}-1\right)-\frac{a\left|f^{\prime}(b)\right|}{(\alpha+3) 2^{\alpha+3}} \\
& -\frac{b\left|f^{\prime}(a)\right|+a\left|f^{\prime}(b)\right|-2 b\left|f^{\prime}(b)\right|}{(\alpha+2) 2^{\alpha+2}} \\
& \left.-\frac{b\left|f^{\prime}(b)\right|}{(\alpha+1) 2^{\alpha+1}}\right\} \\
& +\frac{b\left[\left|f^{\prime}(a)\right|-\left|f^{\prime}(b)\right|\right]+(a-b)\left|f^{\prime}(b)\right|}{\alpha+2} \\
& \times\left(1-\frac{1}{2^{\alpha+2}}\right)-a\left|f^{\prime}(a)\right| \int_{0}^{1} t^{2}(1-t)^{\alpha} d t \\
& +a\left|f^{\prime}(a)\right| \int_{0}^{1 / 2} t^{2}(1-t)^{\alpha} d t \\
& -b\left|f^{\prime}(a)\right| \int_{0}^{1} t(1-t)^{\alpha+1} d t \\
& +b\left|f^{\prime}(a)\right| \int_{0}^{1 / 2} t(1-t)^{\alpha+1} d t \\
& -a\left|f^{\prime}(b)\right| \int_{0}^{1} t(1-t)^{\alpha+1} d t \\
& \left.+a\left|f^{\prime}(b)\right| \int_{0}^{1 / 2} t(1-t)^{\alpha+1} d t\right\} \\
& +\frac{\ln b-\ln a}{2}\left\{a\left|f^{\prime}(a)\right| \int_{0}^{1 / 2} t^{2}(1-t)^{\alpha} d t\right. \\
& +b\left|f^{\prime}(a)\right| \int_{0}^{1 / 2} t(1-t)^{\alpha+1} d t \\
& +a\left|f^{\prime}(b)\right| \int_{0}^{1 / 2} t(1-t)^{\alpha+1} d t \\
& +\frac{b\left|f^{\prime}(b)\right|}{\alpha+3}\left(\frac{1}{2^{\alpha+3}}-1\right)-\frac{a\left|f^{\prime}(b)\right|}{(\alpha+3) 2^{\alpha+3}} \\
& -\frac{b\left|f^{\prime}(a)\right|+a\left|f^{\prime}(b)\right|-2 b\left|f^{\prime}(b)\right|}{(\alpha+2) 2^{\alpha+2}} \\
& \left.-\frac{b\left|f^{\prime}(b)\right|}{(\alpha+1) 2^{\alpha+1}}\right\} \\
& \leq \frac{\left|f^{\prime}(b)\right|-\left|f^{\prime}(a)\right|}{2} \\
& +\frac{\ln b-\ln a}{2} \\
& \times\left\{\left(1-\frac{1}{2^{\alpha+1}}\right)\right.
\end{aligned}
$$




$$
\begin{aligned}
& \times\left[\frac{b\left|f^{\prime}(b)\right|}{\alpha+1}+\frac{b\left|f^{\prime}(a)\right|+a\left|f^{\prime}(b)\right|-2 b\left|f^{\prime}(b)\right|}{\alpha+2}\right] \\
& +\frac{a\left|f^{\prime}(a)\right|-a\left|f^{\prime}(b)\right|-b\left|f^{\prime}(a)\right|}{\alpha+3} \\
& -\frac{a\left|f^{\prime}(a)\right|-b\left|f^{\prime}(a)\right|}{(\alpha+3) 2^{\alpha+3}}+\frac{2 a\left|f^{\prime}(a)\right|}{(\alpha+1)(\alpha+2)(\alpha+3)} \\
& +\frac{b\left|f^{\prime}(a)\right|+a\left|f^{\prime}(b)\right|-2 a\left|f^{\prime}(a)\right|}{(\alpha+1)(\alpha+3)} \\
& +\frac{2 a\left(\left|f^{\prime}(a)\right|-\left|f^{\prime}(b)\right|\right)-2 b\left|f^{\prime}(a)\right|}{2^{\alpha+3}(\alpha+2)(\alpha+3)} \\
& +\frac{b\left|f^{\prime}(a)\right|+a\left|f^{\prime}(b)\right|}{2^{\alpha+2}(\alpha+2)} \\
& \left.+\frac{a\left|f^{\prime}(a)\right|}{(\alpha+1) 2^{\alpha+2}}-\frac{a\left|f^{\prime}(a)\right|}{(\alpha+2)^{2} 2^{\alpha+2}}\right\}
\end{aligned}
$$

The proof is done.

Theorem 15. Let $f:[0, b] \rightarrow \mathbb{R}^{+}$be a differentiable mapping. If $\left|f^{\prime}\right|^{q}$ is measurable and $\left|f^{\prime}\right|^{q},(q>1)$ is GG-convex on $[a, b]$, for some fired $\alpha \in(0, \infty)$ and $t \in[0,1], 0 \leq a<b$, then the following integrals hold:

$$
\begin{gathered}
\left|\frac{\Gamma(\alpha+1)}{2(\ln b-\ln a)^{\alpha}}\left[{ }_{H} J_{a^{+}}^{\alpha} f(b)+{ }_{H} J_{b^{-}}^{\alpha} f(a)\right]-f\left(\frac{a+b}{2}\right)\right| \\
\leq \frac{\left|f^{\prime}(b)\right|-\left|f^{\prime}(a)\right|}{2}+\frac{\ln b-\ln a}{2} \\
\quad \times\left(\frac{\left|f^{\prime}(a)\right|^{q}+2\left|f^{\prime}(a)\right|^{q}-\left|f^{\prime}(a)\right|^{q}}{2}\right)^{1 / q} \\
\quad \times\left(\frac{a^{p}-2 b^{p}}{p \alpha+2}+\frac{b^{p}}{p \alpha+1}-\frac{2 b^{p}+a^{p}}{2^{p \alpha+2}(p \alpha+1)}\right. \\
\quad+\frac{2 b^{p}-a^{p}}{2^{p \alpha+2}(p \alpha+2)}-\frac{a^{p}}{p \alpha(p \alpha+1)} \\
\quad-\frac{a^{p}+b^{p}}{2^{p \alpha+2}(p \alpha+1)(p \alpha+2)} \\
\left.\quad+\frac{2 a^{p}}{(p \alpha+1)(p \alpha+2)}\right)^{1 / p}
\end{gathered}
$$

where $1 / p+1 / q=1$
Proof. By using Definition 4 and Lemmas 2 and 6, we have

$$
\begin{aligned}
&\left|\frac{\Gamma(\alpha+1)}{2(\ln b-\ln a)^{\alpha}}\left[{ }_{H} J_{a^{+}}^{\alpha} f(b)+{ }_{H} J_{b^{-}}^{\alpha} f(a)\right]-f\left(\frac{a+b}{2}\right)\right| \\
& \leq \frac{b-a}{2} \int_{0}^{1}\left|k f^{\prime}(t a+(1-t) b)\right| d t \\
& \quad+\frac{\ln b-\ln a}{2} \int_{0}^{1} a^{t} b^{1-t}\left|(1-t)^{\alpha}-t^{\alpha}\right|\left|f^{\prime}\left(a^{t} b^{1-t}\right)\right| d t \\
& \leq \frac{b-a}{2} \int_{0}^{1}|k|\left|f^{\prime}(a t+b(1-t))\right| d t \\
&+\frac{\ln b-\ln a}{2} \int_{0}^{1} a^{t} b^{1-t}\left|(1-t)^{\alpha}-t^{\alpha}\right|\left|f^{\prime}\left(a^{t} b^{1-t}\right)\right| d t \\
& \leq \frac{(b-a)}{2} \int_{0}^{1}\left|f^{\prime}(a t+b(1-t))\right| d t \\
&+\frac{\ln b-\ln a}{2} \int_{0}^{1} a^{t} b^{1-t}\left|(1-t)^{\alpha}-t^{\alpha}\right|\left|f^{\prime}\left(a^{t} b^{1-t}\right)\right| d t \\
& \leq \frac{-1}{2}\left(\left|f^{\prime}(a)\right|-\left|f^{\prime}(b)\right|\right) \\
&+\frac{\ln b-\ln a}{2}\left[\int_{1 / 2}^{1}\left[t a^{p}+(1-t) b^{p}\right]\left(t^{p a}-(1-t)^{p a}\right) d t\right. \\
&\left.+\frac{\ln b-\ln a}{2}\left(\int_{0}^{1}\left[t\left|f^{\prime}(a)\right|^{q}+\left.(1-t)\left|f^{\prime} b^{1-t}\right|(b)\right|^{q}\right] d t\right)^{1 / q}(a)|-| f^{\prime}(b) \mid\right) \\
&\left.\left.\times\left(\int_{0}^{1}\left[t a^{p}+(1-t) b^{p}\right]\left|(1-t)^{\alpha}-t^{\alpha}\right|\right)^{p} d t\right)^{p} d t\right)^{1 / p} \\
& \leq \frac{-1}{2}\left(\left|f^{\prime}(a)\right|-\left|f^{\prime}(b)\right|\right) \\
&+\left(\int_{0}^{1}\left|f^{\prime}\left(a^{t} b^{1-t}\right)\right|^{q} d t\right) \\
& \leq \frac{-1}{2}\left(\left|f^{\prime}(a)\right|-\left|f^{\prime}(b)\right|\right) \\
&+\frac{\ln b-\ln a}{2}\left(\int_{0}^{1} a^{p t} b^{p(1-t)}\left|(1-t)^{\alpha}-t^{\alpha}\right|^{p} d t\right)^{1 / p} \\
&
\end{aligned}
$$




$$
\begin{aligned}
& \left.+\int_{0}^{1 / 2}\left[t a^{p}+(1-t) b^{p}\right]\left[(1-t)^{p \alpha}-t^{p \alpha}\right] d t\right)^{1 / p} \\
& \times\left(\frac{\left|f^{\prime}(a)\right|^{q}}{2}+\left|f^{\prime}(b)\right|^{q}-\frac{\left|f^{\prime}(b)\right|^{q}}{2}\right)^{1 / q} \\
& \leq \frac{-1}{2}\left(\left|f^{\prime}(a)\right|-\left|f^{\prime}(b)\right|\right) \\
& +\frac{\ln b-\ln a}{2}\left(\frac{\left|f^{\prime}(a)\right|^{q}+2\left|f^{\prime}(b)\right|^{q}-\left|f^{\prime}(b)\right|^{q}}{2}\right)^{1 / q} \\
& \times\left(a^{p} \int_{1 / 2}^{1} t^{p \alpha+1} d t-a^{p} \int_{1 / 2}^{1} t(1-t)^{p \alpha} d t\right. \\
& +b^{p} \int_{1 / 2}^{1} t^{p \alpha}(1-t) d t-b^{p} \int_{1 / 2}^{1}(1-t)^{p \alpha+1} d t \\
& +a^{p} \int_{0}^{1 / 2} t(1-t)^{p \alpha} d t-a^{p} \int_{0}^{1 / 2} t^{p \alpha+1} d t \\
& \left.+b^{p} \int_{0}^{1 / 2}(1-t)^{p \alpha+1}-b^{p} \int_{0}^{1 / 2} t^{p \alpha}(1-t) d t\right)^{1 / p} \\
& \leq \frac{-1}{2}\left(\left|f^{\prime}(a)\right|-\left|f^{\prime}(b)\right|\right) \\
& +\frac{\ln b-\ln a}{2}\left(\frac{\left|f^{\prime}(a)\right|^{q}+2\left|f^{\prime}(b)\right|^{q}-\left|f^{\prime}(b)\right|^{q}}{2}\right)^{1 / q} \\
& \times\left(\frac{a^{p}}{p \alpha+2}-a^{p} \int_{0}^{1} t(1-t)^{p \alpha-1} d t\right. \\
& +a^{p} \int_{0}^{1 / 2} t(1-t)^{p \alpha} d t+\frac{b^{p}}{p \alpha+1}-\frac{1}{2^{p \alpha+1}} \frac{b^{p}}{p \alpha+1} \\
& -\frac{b^{p}}{p \alpha+2}+\frac{1}{2^{p \alpha+2}} \frac{b^{p}}{p \alpha+2}+a^{p} \int_{0}^{1 / 2} t(1-t)^{p \alpha} d t \\
& -\frac{1}{2^{p \alpha+2}} \frac{a^{p}}{p \alpha+2}+\frac{1}{2^{p \alpha+2}} \frac{b^{p}}{p \alpha+2}-\frac{b^{p}}{p \alpha+2} \\
& \left.-b^{p} \int_{0}^{1 / 2} t^{p \alpha}(1-t) d t\right)^{1 / p} \\
& \leq \frac{\left|f^{\prime}(b)\right|-\left|f^{\prime}(a)\right|}{2} \\
& +\frac{\ln b-\ln a}{2}\left(\frac{\left|f^{\prime}(a)\right|^{q}+2\left|f^{\prime}(b)\right|^{q}-\left|f^{\prime}(b)\right|^{q}}{2}\right)^{1 / q} \\
& \times\left(\frac{a^{p}-2 b^{p}}{p \alpha+2}+\frac{b^{p}}{p \alpha+1}-\frac{2 b^{p}+a^{p}}{2^{p \alpha+2}(p \alpha+1)}\right.
\end{aligned}
$$$$
+\frac{2 b^{p}-a^{p}}{2^{p \alpha+2}(p \alpha+2)}-\frac{a^{p}}{p \alpha(p \alpha+1)}
$$$$
\begin{aligned}
& -\frac{a^{p}+b^{p}}{2^{p \alpha+2}(p \alpha+1)(p \alpha+2)} \\
& \left.+\frac{2 a^{p}}{(p \alpha+1)(p \alpha+2)}\right)^{1 / p} .
\end{aligned}
$$

The proof is done.

Theorem 16. Let $f:[0, b] \rightarrow \mathbb{R}^{+}$be a differentiable mapping. If $\left|f^{\prime}\right|$ is measurable and $\left|f^{\prime}\right|$ is GG-convex on $[a, b]$, for some fired $\alpha \in(0, \infty)$ and $t \in[0,1], 0 \leq a<b$, then the following integrals hold:

$$
\begin{aligned}
& \left|\frac{\Gamma(\alpha+1)}{2(\ln b-\ln a)^{\alpha}}\left[{ }_{H} J_{a^{+}}^{\alpha} f(b)+{ }_{H} J_{b^{-}}^{\alpha} f(a)\right]-f\left(\frac{a+b}{2}\right)\right| \\
& \leq \frac{1}{2}\left(\left|f^{\prime}(b)\right|-\left|f^{\prime}(a)\right|\right) \\
& +b\left|f^{\prime}(b)\right| \frac{\ln b-\ln a}{2} \\
& \times\left\{\frac{a\left|f^{\prime}(a)\right|}{b\left|f^{\prime}(b)\right|} \sum_{i=1}^{\infty}(-1)^{i-1} \frac{\left[\ln \left(a\left|f^{\prime}(a)\right| / b\left|f^{\prime}(b)\right|\right)\right]^{i-1}}{(\alpha+1)_{i}}\right. \\
& +\sum_{i=1}^{\infty} \frac{\left[\ln \left(a\left|f^{\prime}(a)\right| / b\left|f^{\prime}(b)\right|\right)\right]^{i-1}}{(\alpha+1)_{i}} \\
& -\frac{(1 / 2)^{\alpha-1}\left(a\left|f^{\prime}(a)\right| / b\left|f^{\prime}(b)\right|\right)^{1 / 2}}{\alpha+1} \\
& \left.+2 \frac{1-(1 / 2)^{\alpha}\left(a\left|f^{\prime}(a)\right| / b\left|f^{\prime}(b)\right|\right)^{1 / 2}}{\ln \left(a\left|f^{\prime}(a)\right| / b\left|f^{\prime}(b)\right|\right)}\right\} .
\end{aligned}
$$

Proof. By using Definition 4 and Lemmas 2, 7, 8, and 9, we have

$$
\begin{gathered}
\left|\frac{\Gamma(\alpha+1)}{2(\ln b-\ln a)^{\alpha}}\left[{ }_{H} J_{a^{+}}^{\alpha} f(b)+{ }_{H} J_{b^{-}}^{\alpha} f(a)\right]-f\left(\frac{a+b}{2}\right)\right| \\
\leq\left|\frac{b-a}{2} \int_{0}^{1} k f^{\prime}(t a+(1-t) b) d t\right| \\
+\frac{\ln b-\ln a}{2} \int_{0}^{1} a^{t} b^{1-t}\left|(1-t)^{\alpha}-t^{\alpha}\right| \\
\times\left|f^{\prime}\left(a^{t} b^{1-t}\right)\right| d t
\end{gathered}
$$




$$
\begin{aligned}
& \leq \frac{-1}{2}(|f(a)|-|f(b)|) \\
& +\frac{\ln b-\ln a}{2} \\
& \times\left\{\int_{1 / 2}^{1} a^{t} b^{1-t}\left[t^{\alpha}-(1-t)^{\alpha}\right]\left|f^{\prime}\left(a^{t} b^{1-t}\right)\right| d t\right. \\
& \left.+\int_{0}^{1 / 2} a^{t} b^{1-t}\left[(1-t)^{\alpha}-t^{\alpha}\right]\left|f^{\prime}\left(a^{t} b^{1-t}\right)\right| d t\right\} \\
& \leq \frac{-1}{2}(|f(a)|-|f(b)|) \\
& +\frac{\ln b-\ln a}{2} \\
& \times \int_{1 / 2}^{1} a^{t} b^{1-t}\left[t^{\alpha}-(1-t)^{\alpha}\right]\left|f^{\prime}(a)\right|^{t}\left|f^{\prime}(b)\right|^{1-t} d t \\
& +\frac{\ln b-\ln a}{2} \\
& \times \int_{0}^{1 / 2} a^{t} b^{1-t}\left[(1-t)^{\alpha}-t^{\alpha}\right]\left|f^{\prime}(a)\right|^{t}\left|f^{\prime}(b)\right|^{1-t} d t \\
& \leq \frac{-1}{2}(|f(a)|-|f(b)|) \\
& +b\left|f^{\prime}(b)\right| \frac{\ln b-\ln a}{2} \\
& \times \int_{1 / 2}^{1}\left(\frac{a\left|f^{\prime}(a)\right|}{b\left|f^{\prime}(b)\right|}\right)^{t}\left[t^{\alpha}-(1-t)^{\alpha}\right] d t \\
& +b\left|f^{\prime}(b)\right| \frac{\ln b-\ln a}{2} \\
& \times \int_{0}^{1 / 2}\left(\frac{a\left|f^{\prime}(a)\right|}{b\left|f^{\prime}(b)\right|}\right)^{t}\left[(1-t)^{\alpha}-t^{\alpha}\right] d t \\
& \leq \frac{1}{2}(|f(b)|-|f(a)|) \\
& +b\left|f^{\prime}(b)\right| \frac{\ln b-\ln a}{2} \\
& \times\left\{\int_{0}^{1}\left(\frac{a\left|f^{\prime}(a)\right|}{b\left|f^{\prime}(b)\right|}\right)^{t} t^{\alpha} d t\right. \\
& -\int_{0}^{1}\left(\frac{a\left|f^{\prime}(a)\right|}{b\left|f^{\prime}(b)\right|}\right)^{t}(1-t)^{\alpha} d t \\
& -2 \int_{0}^{1 / 2}\left(\frac{a\left|f^{\prime}(a)\right|}{b\left|f^{\prime}(b)\right|}\right)^{t} t^{\alpha} d t \\
& \left.+2 \int_{0}^{1 / 2}\left(\frac{a\left|f^{\prime}(a)\right|}{b\left|f^{\prime}(b)\right|}\right)^{t}(1-t)^{\alpha} d t\right\}
\end{aligned}
$$$$
\leq \frac{1}{2}(|f(b)|-|f(a)|)
$$$$
\begin{aligned}
& +b\left|f^{\prime}(b)\right| \frac{\ln b-\ln a}{2} \\
& \times\left\{\frac{a\left|f^{\prime}(a)\right|}{b\left|f^{\prime}(b)\right|} \sum_{i=1}^{\infty}(-1)^{i-1} \frac{\left[\ln \left(a\left|f^{\prime}(a)\right| / b\left|f^{\prime}(b)\right|\right)\right]^{i-1}}{(\alpha+1)_{i}}\right.
\end{aligned}
$$$$
-\sum_{i=1}^{\infty} \frac{\left[\ln \left(a\left|f^{\prime}(a)\right| / b\left|f^{\prime}(b)\right|\right)\right]^{i-1}}{(\alpha+1)_{i}}
$$$$
-\left(\frac{1}{2}\right)^{\alpha-1}\left(\frac{a\left|f^{\prime}(a)\right|}{b\left|f^{\prime}(b)\right|}\right)^{1 / 2}
$$$$
\times \sum_{i=1}^{\infty} \frac{\left[-(1 / 2) \ln \left(a\left|f^{\prime}(a)\right| / b\left|f^{\prime}(b)\right|\right)\right]^{i-1}}{(\alpha+1)_{i}}
$$$$
+2 \sum_{i=0}^{\infty} \frac{\left[\ln \left(a\left|f^{\prime}(a)\right| / b\left|f^{\prime}(b)\right|\right)\right]^{i-1}}{(\alpha+1)_{i}}
$$$$
\left.\times\left[1-\left(\frac{1}{2}\right)^{\alpha+i}\left(\frac{a\left|f^{\prime}(a)\right|}{b\left|f^{\prime}(b)\right|}\right)^{1 / 2}\right]\right\}
$$$$
\leq \frac{1}{2}\left(\left|f^{\prime}(b)\right|-\left|f^{\prime}(a)\right|\right)
$$$$
+b\left|f^{\prime}(b)\right| \frac{\ln b-\ln a}{2}
$$$$
\times\left\{\frac{a\left|f^{\prime}(a)\right|^{\infty}}{b\left|f^{\prime}(b)\right|_{i=1}^{\infty}(-1)^{i-1}} \frac{\left[\ln \left(a\left|f^{\prime}(a)\right| / b\left|f^{\prime}(b)\right|\right)\right]^{i-1}}{(\alpha+1)_{i}}\right.
$$$$
+\sum_{i=1}^{\infty} \frac{\left[\ln \left(a\left|f^{\prime}(a)\right| / b\left|f^{\prime}(b)\right|\right)\right]^{i-1}}{(\alpha+1)_{i}}
$$$$
-\frac{(1 / 2)^{\alpha-1}\left(a\left|f^{\prime}(a)\right| / b\left|f^{\prime}(b)\right|\right)^{1 / 2}}{\alpha+1}
$$$$
\left.+2 \frac{1-(1 / 2)^{\alpha}\left(a\left|f^{\prime}(a)\right| / b\left|f^{\prime}(b)\right|\right)^{1 / 2}}{\ln \left(a\left|f^{\prime}(a)\right| / b\left|f^{\prime}(b)\right|\right)}\right\} .
$$

The proof is done.

Theorem 17. Let $f:[0, b] \rightarrow \mathbb{R}^{+}$be a differentiable mapping. If $\left|f^{\prime}\right|^{q}$ is measurable and $\left|f^{\prime}\right|^{q},(q>1)$ is GG-convex on $[a, b]$, 
International Journal of Mathematics and Mathematical Sciences

15

for some fired $\alpha \in(0, \infty)$ and $t \in[0,1], 0 \leq a<b$, then the following integrals hold:

$$
\begin{aligned}
\left|\frac{\Gamma(\alpha+1)}{2(\ln b-\ln a)^{\alpha}}\left[{ }_{H} J_{a^{+}}^{\alpha} f(b)+{ }_{H} J_{b^{-}}^{\alpha} f(a)\right]-f\left(\frac{a+b}{2}\right)\right| & \frac{1}{2}(|f(b)|-|f(a)|) \\
+ & \frac{\ln b-\ln a}{2} \\
& \times\left(a^{p} \sum_{i=1}^{\infty}(-1)^{i-1} \frac{\left[(1 / 2) \ln (a / b)^{p}\right]^{i-1}}{(p \alpha+1)_{i}}\right. \\
& +b^{p} \sum_{i=1}^{\infty} \frac{\left[\ln (a / b)^{p}\right]^{i-1}}{(p \alpha+1)_{i}}-\frac{(1 / 2)^{p \alpha-1}\left(a b^{p / 2}\right)}{p \alpha+1} \\
& \left.\quad-\left[\ln \left(\frac{a}{b}\right)^{p}\right]^{-1}\left[\left(\frac{1}{2}\right)^{p \alpha-1}(a b)^{p / 2}+2 b^{p}\right]\right)^{1 / p} \\
& \quad\left(\frac{\ln \left(\left|f^{\prime}(a)\right| /\left|f^{\prime}(b)\right|\right)^{q}}{\left(\left|f^{\prime}\right|^{q}\right.}\left[\left.\frac{f^{\prime}(a)}{f^{\prime}(b)}\right|^{q}-1\right]\right)^{1 / q}
\end{aligned}
$$

where $1 / p+1 / q=1$.

Proof. By using Definition 4 and Lemmas 2, 7, 8, and 9, we have

$$
\begin{aligned}
&\left|\frac{\Gamma(\alpha+1)}{2(\ln b-\ln a)^{\alpha}}\left[{ }_{H} J_{a^{+}}^{\alpha} f(b)+{ }_{H} J_{b^{-}}^{\alpha} f(a)\right]-f\left(\frac{a+b}{2}\right)\right| \\
& \leq \frac{b-a}{2} \int_{0}^{1}\left|k f^{\prime}(t a+(1-t) b)\right| d t \\
& \quad+\frac{\ln b-\ln a}{2} \int_{0}^{1} a^{t} b^{1-t}\left|(1-t)^{\alpha}-t^{\alpha}\right|\left|f^{\prime}\left(a^{t} b^{1-t}\right)\right| d t \\
& \leq \frac{b-a}{2} \int_{0}^{1}|k|\left|f^{\prime}(t a+(1-t) b)\right| d t \\
&+\frac{\ln b-\ln a}{2} \int_{0}^{1} a^{t} b^{1-t}\left|(1-t)^{\alpha}-t^{\alpha}\right|\left|f^{\prime}\left(a^{t} b^{1-t}\right)\right| d t \\
& \leq \frac{b-a}{2} \int_{0}^{1}|k|\left|f^{\prime}(t a+(1-t) b)\right| d t \\
&+\frac{\ln b-\ln a}{2}\left(\int_{0}^{1}\left(a^{t} b^{1-t}\left|(1-t)^{\alpha}-t^{\alpha}\right|\right)^{p} d t\right)^{1 / p} \\
& \times\left(\int_{0}^{1}\left|f^{\prime}\left(a^{t} b^{1-t}\right)\right|^{q} d t\right)^{1 / q}
\end{aligned}
$$

$$
\begin{aligned}
& \leq \frac{-1}{2}(|f(a)|-|f(b)|) \\
& +\frac{\ln b-\ln a}{2} \\
& \times\left(b^{p} \int_{1 / 2}^{1}\left(\frac{a}{b}\right)^{p t} t^{p \alpha} d t-b^{p} \int_{1 / 2}^{1}\left(\frac{a}{b}\right)^{p t}(1-t)^{p \alpha} d t\right. \\
& \left.+b^{p} \int_{0}^{1 / 2}\left(\frac{a}{b}\right)^{p t}(1-t)^{p \alpha} d t-b^{p} \int_{0}^{1 / 2}\left(\frac{a}{b}\right)^{p t} t^{p \alpha} d t\right)^{1 / p} \\
& \times\left(\left|f^{\prime}(b)\right|^{q} \int_{0}^{1}\left[\frac{\left|f^{\prime}(a)\right|}{\left|f^{\prime}(b)\right|}\right]^{q t} d t\right)^{1 / q} \\
& \leq \frac{-1}{2}(|f(a)|-|f(b)|) \\
& +\frac{\ln b-\ln a}{2} \\
& \times\left(b^{p} \int_{0}^{1}\left(\frac{a}{b}\right)^{p t} t^{p \alpha} d t-b^{p} \int_{0}^{1 / 2}\left(\frac{a}{b}\right)^{p t} t^{p \alpha} d t\right. \\
& -b^{p} \int_{0}^{1}\left(\frac{a}{b}\right)^{p t}(1-t)^{p \alpha} d t+\int_{0}^{1 / 2}\left(\frac{a}{b}\right)^{p t}(1-t)^{p \alpha} d t \\
& \left.+b^{p} \int_{0}^{1 / 2}\left(\frac{a}{b}\right)^{p t}(1-t)^{p \alpha} d t-b^{p} \int_{0}^{1 / 2}\left(\frac{a}{b}\right)^{p t} t^{p \alpha} d t\right)^{1 / p} \\
& \times\left(\frac{\left|f^{\prime}(b)\right|^{q}}{\ln \left(\left|f^{\prime}(a)\right| /\left|f^{\prime}(b)\right|\right)^{q}}\left[\left|\frac{f^{\prime}(a)}{f^{\prime}(b)}\right|^{q}-1\right]\right)^{1 / q} \\
& \leq \frac{-1}{2}(|f(a)|-|f(b)|) \\
& +\frac{\ln b-\ln a}{2} \\
& \times\left(b^{p}\left(\frac{a}{b}\right)^{p} \sum_{i=1}^{\infty}(-1)^{i-1} \frac{\left[\ln (a / b)^{p}\right]^{i-1}}{(p \alpha+1)_{i}}\right. \\
& -b^{p} \sum_{i=0}^{\infty} \frac{\left[\ln (a / b)^{p}\right]^{i-1}}{(p \alpha+1)_{i}}-2 b^{p} \int_{0}^{1 / 2}\left(\frac{a}{b}\right)^{p t} t^{p \alpha} d t \\
& \left.+2 b^{p} \int_{0}^{1 / 2}\left(\frac{a}{b}\right)^{p t}(1-t)^{p \alpha} d t\right)^{1 / p} \\
& \times\left(\frac{\left|f^{\prime}(b)\right|^{q}}{\ln \left(\left|f^{\prime}(a)\right| /\left|f^{\prime}(b)\right|\right)^{q}}\left[\left|\frac{f^{\prime}(a)}{f^{\prime}(b)}\right|^{q}-1\right]\right)^{1 / q} \\
& \leq \frac{-1}{2}(|f(a)|-|f(b)|) \\
& +\frac{\ln b-\ln a}{2} \\
& \times\left(b^{p}\left(\frac{a}{b}\right)^{p} \sum_{i=1}^{\infty}(-1)^{i-1} \frac{\left[\ln (a / b)^{p}\right]^{i-1}}{(p \alpha+1)_{i}}\right.
\end{aligned}
$$




$$
\begin{aligned}
& -b^{p} \sum_{i=1}^{\infty} \frac{\left[\ln (a / b)^{p}\right]^{i-1}}{(p \alpha+1)_{i}} \\
& -\left(\frac{1}{2}\right)^{p \alpha} b^{p}\left(\frac{a}{b}\right)^{p / 2} \sum_{i=1}^{\infty} \frac{\left[-(1 / 2) \ln (a / b)^{p}\right]^{i-1}}{(p \alpha+1)_{i}} \\
& \left.+2 b^{p} \sum_{i=0}^{\infty} \frac{\left[\ln (a / b)^{p}\right]^{i-1}}{(p \alpha+1)_{i}}\left[1-\left(\frac{a}{b}\right)^{p / 2}\left(1-\frac{1}{2}\right)^{p \alpha+i}\right]\right)^{1 / p} \\
& \times\left(\frac{\left|f^{\prime}(b)\right|^{q}}{\ln \left(\left|f^{\prime}(a)\right| /\left|f^{\prime}(b)\right|\right)^{q}}\left[\left|\frac{f^{\prime}(a)}{f^{\prime}(b)}\right|^{q}-1\right]\right)^{1 / q} \\
& \leq \frac{-1}{2}(|f(a)|-|f(b)|) \\
& +\frac{\ln b-\ln a}{2} \\
& \times\left(a^{p} \sum_{i=1}^{\infty}(-1)^{i-1} \frac{\left[\ln (a / b)^{p}\right]^{i-1}}{(p \alpha+1)_{i}}-b^{p} \sum_{i=1}^{\infty} \frac{\left[\ln (a / b)^{p}\right]^{i-1}}{(p \alpha+1)_{i}}\right. \\
& -\left(\frac{1}{2}\right)^{p \alpha}(a b)^{p / 2} \sum_{i=1}^{\infty} \frac{\left[-(1 / 2) \ln (a / b)^{p}\right]^{i-1}}{(p \alpha+1)_{i}} \\
& +2 b^{p} \sum_{i=0}^{\infty} \frac{\left[\ln (a / b)^{p}\right]^{i-1}}{(p \alpha+1)_{i}} \\
& \left.-2 b^{p}\left(\frac{a}{b}\right)^{p / 2} \sum_{i=0}^{\infty}\left(1-\frac{1}{2}\right)^{p \alpha+i} \frac{\left[\ln (a / b)^{p}\right]^{i-1}}{(p \alpha+1)_{i}}\right)^{1 / p} \\
& \times\left(\frac{\left|f^{\prime}(b)\right|^{q}}{\ln \left(\left|f^{\prime}(a)\right| /\left|f^{\prime}(b)\right|\right)^{q}}\left[\left|\frac{f^{\prime}(a)}{f^{\prime}(b)}\right|^{q}-1\right]\right)^{1 / q} \\
& \leq \frac{-1}{2}(|f(a)|-|f(b)|) \\
& +\frac{\ln b-\ln a}{2} \\
& \times\left(a^{p} \sum_{i=1}^{\infty}(-1)^{i-1} \frac{\left[\ln (a / b)^{p}\right]^{i-1}}{(p \alpha+1)_{i}}-b^{p} \sum_{i=1}^{\infty} \frac{\left[\ln (a / b)^{p}\right]^{i-1}}{(p \alpha+1)_{i}}\right. \\
& -\left(\frac{1}{2}\right)^{p \alpha}(a b)^{p / 2} \sum_{i=1}^{\infty} \frac{\left[-(1 / 2) \ln (a / b)^{p}\right]^{i-1}}{(p \alpha+1)_{i}} \\
& +2 b^{p} \sum_{i=1}^{\infty} \frac{\left[\ln (a / b)^{p}\right]^{i-1}}{(p \alpha+1)_{i}}+2 b^{p}\left[\ln \left(\frac{a}{b}\right)^{p}\right]^{-1} \\
& \left.-2 b^{p}\left(\frac{a}{b}\right)^{p / 2} \sum_{i=0}^{\infty}\left(1-\frac{1}{2}\right)^{p \alpha+i} \frac{\left[\ln (a / b)^{p}\right]^{i-1}}{(p \alpha+1)_{i}}\right)^{1 / p} \\
& \times\left(\frac{\left|f^{\prime}(b)\right|^{q}}{\ln \left(\left|f^{\prime}(a)\right| /\left|f^{\prime}(b)\right|\right)^{q}}\left[\left|f^{\prime}(a) / f^{\prime}(b)\right|^{q}-1\right]\right)^{1 / q}
\end{aligned}
$$

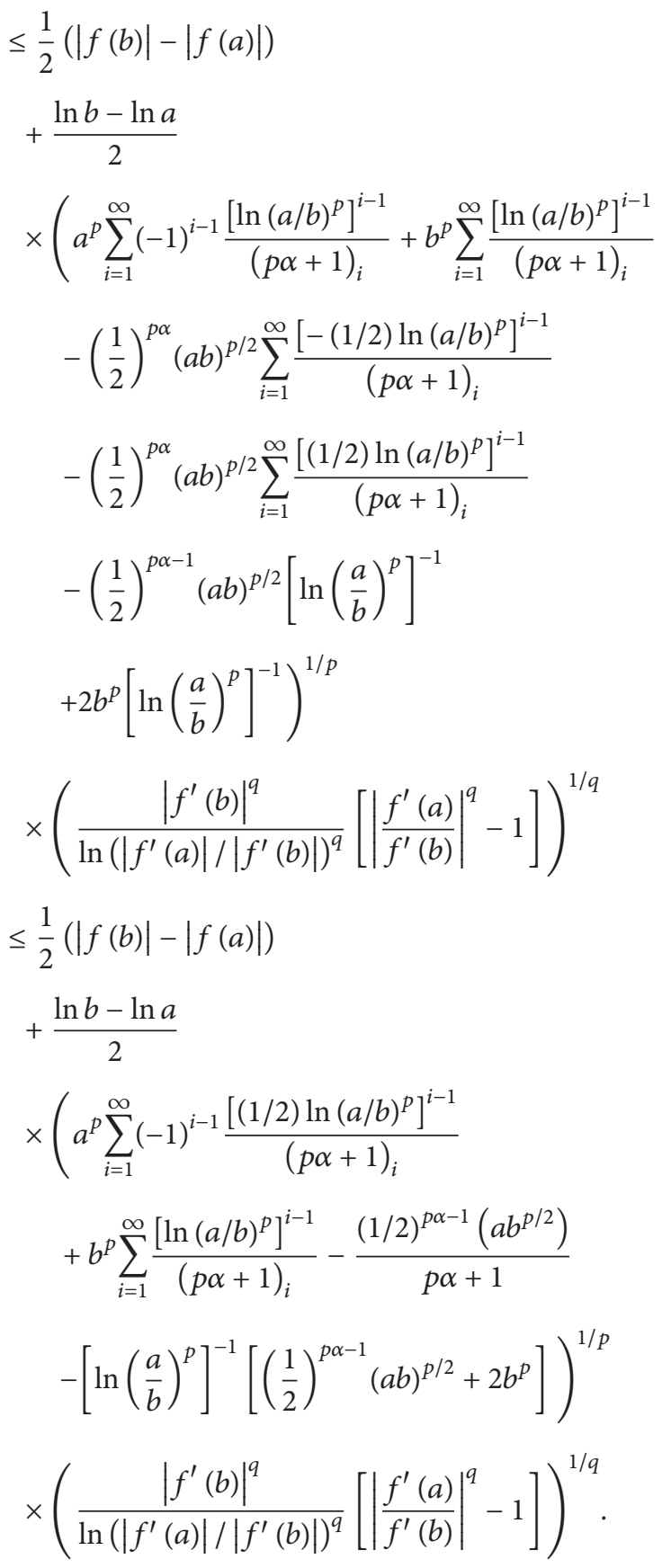

The proof is done.

\section{Applications to Special Means}

Consider the following special means (see [23]) for arbitrary real numbers $x, y, x \neq y$ as follows:

$$
\begin{aligned}
& \left(\mathrm{M}_{1}\right) A(x, y)=(x+y) / 2, x, y \in \mathbb{R} \\
& \left(\mathrm{M}_{2}\right) H(x, y)=2 /(1 / x+1 / y), x, y \in \mathbb{R} \backslash\{0\} \\
& \left(\mathrm{M}_{3}\right) G(x, y)=\sqrt{x y} \\
& \left(\mathrm{M}_{4}\right) L(x, y)=(y-x) /(\ln |y|-\ln |x|),|x| \neq|y|, x y \neq 0
\end{aligned}
$$




$$
\begin{aligned}
\left(\mathrm{M}_{5}\right) & L_{n}(x, y)=\left[\left(y^{n+1}-x^{n+1}\right) /(n+1)(y-x)\right]^{1 / n}, n \in \mathbb{Z} \backslash \\
& \{-1,0\}, x, y \in \mathbb{R}, x \neq y .
\end{aligned}
$$

We give some applications to special means of real numbers.

Proposition 18. Let $a, b \in \mathbb{R}^{+} \backslash\{0\}, 0 \leq a<b, x \in[0, b]$. Then,

$$
\begin{aligned}
& |A(a, b)-L(a, b)| \leq \frac{\ln b-\ln a}{2} \frac{63 a-b}{192} ; \\
& |A(a, b)-L(a, b)| \leq \frac{\ln b-\ln a}{2}\left(\frac{1}{2}\right)^{1 / q} \\
& \times\left(\frac{a^{p}-2 b^{p}}{p+2}+\frac{b^{p}}{p+1}-\frac{2 b^{p}+a^{p}}{2^{p+1}(p+1)}\right. \\
& -\frac{b^{p}-a^{p}}{2^{p+2}(p+2)}+\frac{a^{p}}{(p+1)(p+2)} \\
& \left.-\frac{a^{p}}{2^{p+1}(p+1)(p+2)}\right)^{1 / p} ; \\
& |L(a, b)-A(a, b)| \leq \frac{\ln b-\ln a}{2} \frac{63 a-b}{192} ; \\
& |L(a, b)-A(a, b)| \leq \frac{\ln b-\ln a}{2}\left(\frac{1}{2}\right)^{1 / q} \\
& \times\left(\frac{a^{p}-2 b^{p}}{p+2}+\frac{b^{p}}{p+1}-\frac{2 b^{p}+a^{p}}{2^{p+1}(p+1)}\right. \\
& -\frac{b^{p}-a^{p}}{2^{p+2}(p+2)}+\frac{a^{p}}{(p+1)(p+2)} \\
& \left.-\frac{a^{p}}{2^{p+1}(p+1)(p+2)}\right)^{1 / p} ; \\
& |A(a, b)-L(a, b)| \leq b \frac{\ln b-\ln a}{2} \\
& \times\left[\frac{a / b-1}{\ln (a / b)}\right. \\
& \left.+2 \frac{(a / b)^{1 / 2}-a / b+(a / b)^{2}-1}{(\ln (a / b))^{2}}\right] .
\end{aligned}
$$

By using Lemmas 7, 8, and 9, we have

$$
\begin{aligned}
|A(a, b)-L(a, b)| \leq & b \frac{\ln b-\ln a}{2} \\
\times & \left(I[p+1]-H\left(p+1,\left(\frac{a}{b}\right)^{p}, \frac{1}{2}\right)\right. \\
& -2 J\left(p+1,\left(\frac{a}{b}\right)^{p}\right) \\
& \left.+2 b R\left(p+1,\left(\frac{a}{b}\right)^{p}, \frac{1}{2}\right)\right)^{1 / p} ;
\end{aligned}
$$

$|L(a, b)-A(a, b)| \leq b \frac{\ln b-\ln a}{2}$

$$
\times\left[\frac{a / b-1}{\ln (a / b)}\right.
$$

$$
\left.+2 \frac{(a / b)^{1 / 2}-a / b+(a / b)^{2}-1}{(\ln (a / b))^{2}}\right]
$$

$$
\begin{aligned}
|L(a, b)-A(a, b)| \leq & b \frac{\ln b-\ln a}{2} \\
\times & \left(I[p+1]-H\left(p+1,\left(\frac{a}{b}\right)^{p}, \frac{1}{2}\right)\right. \\
& -2 J\left(p+1,\left(\frac{a}{b}\right)^{p}\right) \\
& \left.+2 b R\left(p+1,\left(\frac{a}{b}\right)^{p}, \frac{1}{2}\right)\right)^{1 / p} .
\end{aligned}
$$

Proof. Applying Theorems 10, 11, 14, 15, 12, 13, 16, and 17, for $f(x)=x$ and $\alpha=1$, one can obtain the results immediately.

Proposition 19. Let $a, b \in \mathbb{R}^{+} \backslash\{0\}, 0 \leq a<b, x \in[0, b]$, $n \geq 2$. Then,

$$
\begin{aligned}
& \left|A\left(a^{n}, b^{n}\right)-L_{n-1}^{n-1}(a, b) L(a, b)\right| \\
& \leq \frac{\ln b-\ln a}{2} \frac{22 n a^{n}+12 n b a^{n-1}+9 n a b^{n-1}-12 n b^{n}}{96} ; \\
& \left|A\left(a^{n}, b^{n}\right)-L_{n-1}^{n-1}(a, b) L(a, b)\right| \\
& \leq \frac{\ln b-\ln a}{2}\left(\frac{n^{q} a^{q(n-1)}+n^{q} b^{q(n-1)}}{2}\right)^{1 / q} \\
& \quad \times\left(\frac{a^{p}-2 b^{p}}{p+2}+\frac{b^{p}}{p+1}\right. \\
& \quad-\frac{1}{2^{p+1}} \frac{b^{p}}{p+1}+\frac{1}{2^{p+2}} \frac{b^{p}-a^{p}}{p+2}-\frac{1}{2^{p+3}} \frac{1}{p+3} \\
& \left.\quad-\frac{a^{p}}{p(p+1)}+\frac{2 a^{p}}{(p+1)(p+2)}\right)^{1 / p} ; \\
& \quad+\frac{\ln b-\ln a}{2} \frac{22 n a^{n}+12 n b a^{n-1}+9 n a b^{n-1}-12 n b^{n}}{96} ; \\
& \leq L_{n-1}^{n-1}(a, b) L(a, b)-A^{n}(x, y) \mid \\
& \qquad n-1-n a^{n-1} \\
& 2
\end{aligned}
$$




$$
\begin{aligned}
&\left|L_{n-1}^{n-1}(a, b) L(a, b)-A^{n}(x, y)\right| \\
& \leq \frac{n b^{n-1}-n a^{n-1}}{2} \\
&+ \frac{\ln b-\ln a}{2}\left(\frac{n^{q} a^{q(n-1)}+n^{q} b^{q(n-1)}}{2}\right)^{1 / q} \\
& \times\left(\frac{a^{p}-2 b^{p}}{p+2}+\frac{b^{p}}{p+1}-\frac{1}{2^{p+1}} \frac{b^{p}}{p+1}\right. \\
&+\frac{1}{2^{p+2}} \frac{b^{p}-a^{p}}{p+2}-\frac{1}{2^{p+3}} \frac{1}{p+3} \\
&\left.-\frac{a^{p}}{p(p+1)}+\frac{2 a^{p}}{(p+1)(p+2)}\right)^{1 / p} ; \\
&\left|A\left(a^{n}, b^{n}\right)-L_{n-1}^{n-1}(a, b) L(a, b)\right| n b^{n} \frac{\ln b-\ln a}{2} \\
& \quad \times\left(\frac{a^{n} / b^{n}-1}{\ln \left(a^{n} / b^{n}\right)}+\frac{4\left(a^{n} / b^{n}\right)-2\left(a^{n} / b^{n}\right)-2}{\left(\ln \left(a^{n} / b^{n}\right)\right)^{2}}\right) .
\end{aligned}
$$

By using Lemmas 7, 8, and 9, we have

$$
\begin{aligned}
& \left|A\left(a^{n}, b^{n}\right)-L_{n-1}^{n-1}(a, b) L(a, b)\right| \\
& \leq b \frac{\ln b-\ln a}{2}\left(I[p+1]-H\left(p+1,\left(\frac{a}{b}\right)^{p}, \frac{1}{2}\right)\right. \\
& -2 J\left(p+1,\left(\frac{a}{b}\right)^{p}\right) \\
& \left.+2 b R\left(p+1,\left(\frac{a}{b}\right)^{p}, \frac{1}{2}\right)\right)^{1 / p} \\
& \times\left(\frac{\left|n b^{n-1}\right|^{q}}{\ln \left(a^{n-1} / b^{n-1}\right)^{q}}\left[\left(\frac{a^{n-1}}{b^{n-1}}\right)^{q}-1\right]\right)^{1 / q} ; \\
& \left|L_{n-1}^{n-1}(a, b) L(a, b)-A^{n}(x, y)\right| \\
& \leq \frac{n b^{n-1}-n a^{n-1}}{2} \\
& \quad+n b^{n} \frac{\ln b-\ln a}{2} \\
& \quad \times\left(\frac{a^{n} / b^{n}-1}{\ln \left(a^{n} / b^{n}\right)}+\frac{4\left(a^{n} / b^{n}\right)-2\left(a^{n} / b^{n}\right)-2}{\left(\ln \left(a^{n} / b^{n}\right)\right)^{2}}\right) ;
\end{aligned}
$$

$$
\begin{aligned}
& \left|L_{n-1}^{n-1}(a, b) L(a, b)-A^{n}(x, y)\right| \\
& \leq \frac{n b^{n-1}-n a^{n-1}}{2} \\
& +b \frac{\ln b-\ln a}{2}\left(I[p+1]-H\left(p+1,\left(\frac{a}{b}\right)^{p}, \frac{1}{2}\right)\right. \\
& -2 J\left(p+1,\left(\frac{a}{b}\right)^{p}\right) \\
& \left.+2 b R\left(p+1,\left(\frac{a}{b}\right)^{p}, \frac{1}{2}\right)\right)^{1 / p} \\
& \times\left(\frac{\left|n b^{n-1}\right|^{q}}{\ln \left(a^{n-1} / b^{n-1}\right)^{q}}\left[\left(\frac{a^{n-1}}{b^{n-1}}\right)^{q}-1\right]\right)^{1 / q} .
\end{aligned}
$$

Proof. Applying Theorems 10, 11, 14, 15, 12, 13, 16, and 17 for $f(x)=x^{n}$ and $\alpha=1$, one can obtain the results immediately.

Proposition 20. Let $a, b \in \mathbb{R}^{+} \backslash\{0\}, 0 \leq a<b, x \in[0, b]$, $n \geq 2$. Then,

$$
\begin{aligned}
\left|H^{-1}(a, b)-G^{-2}(a, b) L(a, b)\right| & \\
\leq & \frac{\ln b-\ln a}{2} \\
\times & \left(450(1 / a)+60\left(a / b^{2}\right)-30\left(b / a^{2}\right)\right. \\
& -32(1 / a)+42(1 / a))(1920)^{-1} ; \\
\left|H^{-1}(a, b)-G^{-2}(a, b) L(a, b)\right| & \\
\leq & \frac{\ln b-\ln a}{2}\left(\frac{a^{-2 q}+b^{-2 q}}{2}\right)^{1 / q} \\
\times & \left(\frac{a^{p}-2 b^{p}}{p+2}+\frac{b^{p}}{p+1}-\frac{1}{2^{p+2}} \frac{2 a^{p}+3 b^{p}}{p+1}\right. \\
& +\frac{1}{2^{p+2}} \frac{2 b^{p}-a^{p}}{p+2}-\frac{1}{p(p+1)} \\
& \left.\quad-\frac{2 a^{p}+b^{p}}{2^{p+2}(p+1)(p+2)}\right)^{1 / p} ;
\end{aligned}
$$

$\left|G^{-2}(a, b) L(a, b)-A^{-1}(a, b)\right|$

$$
\begin{aligned}
\leq & \frac{b^{2}-a^{2}}{2 a^{2} b^{2}}+\frac{\ln b-\ln a}{2} \\
\times & \left(450(1 / a)+60\left(a / b^{2}\right)-30\left(b / a^{2}\right)\right. \\
& \quad-32(1 / a)+42(1 / a))(1920)^{-1} ;
\end{aligned}
$$




$$
\begin{aligned}
& \left|G^{-2}(a, b) L(a, b)-A^{-1}(a, b)\right| \\
& \leq \frac{b^{2}-a^{2}}{2 a^{2} b^{2}} \\
& \quad+\frac{\ln b-\ln a}{2}\left(\frac{a^{-2 q}+b^{-2 q}}{2}\right)^{1 / q} \\
& \quad \times\left(\frac{a^{p}-2 b^{p}}{p+2}+\frac{b^{p}}{p+1}-\frac{1}{2^{p+2}} \frac{2 a^{p}+3 b^{p}}{p+1}\right. \\
& \quad+\frac{1}{2^{p+2}} \frac{2 b^{p}-a^{p}}{p+2}-\frac{1}{p(p+1)} \\
& \left.\quad-\frac{2 a^{p}+b^{p}}{2^{p+2}(p+1)(p+2)}\right)^{1 / p} ; \\
& \left|H^{-1}(a, b)-G^{-2}(a, b) L(a, b)\right| \\
& \leq \frac{\ln b-\ln a}{2 b}\left(\frac{b / a-1}{\ln (b / a)}+\frac{4(b / a)^{1 / 2}-2(b / a)-2}{(\ln (b / a))^{2}}\right) .
\end{aligned}
$$

By using Lemmas 7, 8, and 9, we have

$$
\begin{aligned}
& \left|H^{-1}(a, b)-G^{-2}(a, b) L(a, b)\right| \\
& \leq b \frac{\ln b-\ln a}{2}\left(I[p+1]-H\left(p+1,\left(\frac{a}{b}\right)^{p}, \frac{1}{2}\right)\right. \\
& -2 J\left(p+1,\left(\frac{a}{b}\right)^{p}\right) \\
& \left.\quad+2 b R\left(p+1,\left(\frac{a}{b}\right)^{p}, \frac{1}{2}\right)\right)^{1 / p} \\
& \quad \times\left(\frac{\left|1 / b^{2}\right|^{q}}{\ln \left|b^{2} / a^{2}\right|^{q}}\left[\left|\frac{b^{2}}{a^{2}}\right|^{q}-1\right]\right) ; \\
& \left|G^{-2}(a, b) L(a, b)-A^{-1}(a, b)\right| \\
& \leq \frac{b^{2}-a^{2}}{2 a^{2} b^{2}} \\
& \quad+\frac{\ln b-\ln a}{2 b}\left(\frac{b / a-1}{\ln (b / a)}+\frac{4(b / a)^{1 / 2}-2(b / a)-2}{(\ln (b / a))^{2}}\right) ; \\
& +b \frac{\ln b-\ln a}{2}\left(I[p+1]-H\left(p+1,\left(\frac{a}{b}\right)^{p}, \frac{1}{2}\right)\right. \\
& \left|G^{-2}(a, b) L(a, b)-A^{-1}(a, b)\right| \\
& \leq \frac{b^{2}-a^{2}}{2 a^{2} b^{2}}-2 J\left(p+1,\left(\frac{a}{b}\right)^{p}\right)
\end{aligned}
$$

$$
\begin{gathered}
\left.+2 b R\left(p+1,\left(\frac{a}{b}\right)^{p}, \frac{1}{2}\right)\right)^{1 / p} \\
\times\left(\frac{\left|1 / b^{2}\right|^{q}}{\ln \left|b^{2} / a^{2}\right|^{q}}\left[\left|b^{2} / a^{2}\right|^{q}-1\right]\right)^{1 / q} .
\end{gathered}
$$

Proof. Applying Theorems 10, 11, 14, 15, 12, 13, 16, and 17 for $f(x)=1 / x$ and $\alpha=1$, one can obtain the results immediately.

\section{Conflict of Interests}

The authors declare that there is no conflict of interests regarding the publication of this paper.

\section{Acknowledgments}

The authors thank the referees for their careful reading of the paper and insightful comments, which helped to improve the quality of the paper. They would also like to acknowledge the valuable comments and suggestions from the editors, which vastly contributed to improvement of the presentation of the paper. This work was supported in part by Key Project on the Reforms of Teaching Contents and Course System of Guizhou Normal College, Doctor Project of Guizhou Normal College (13BS010), Guizhou Province Education Planning Project (2013A062), and Key Support Subject (Applied Mathematics).

\section{References}

[1] D. Baleanu, J. A. T. Machado, and A. C. J. Luo, Fractional Dynamics and Control, Springer, New York, NY, USA, 2012.

[2] K. Diethelm, The Analysis of Fractional Differential Equations, vol. 2004 of Lecture Notes in Mathematics, Springer, Berlin, Germany, 2010.

[3] A. A. Kilbas, H. M. Srivastava, and J. J. Trujillo, Theory and Applications of Fractional Differential Equations, vol. 204, Elsevier Science B.V., Amsterdam, The Netherlands, 2006.

[4] V. Lakshmikantham, S. Leela, and J. V. Devi, Theory of Fractional Dynamic Systems, Cambridge Scientific, 2009.

[5] K. S. Miller and B. Ross, An Introduction to the Fractional Calculus and Fractional Differential Equations, A Wiley-Interscience Publication, John Wiley \& Sons, New York, NY, USA, 1993.

[6] M. W. Michalski, "Derivatives of noninteger order and their applications," Dissertationes Mathematicae, vol. 328, 47 pages, 1993.

[7] I. Podlubny, Fractional Differential Equations, Academic Press, 1999.

[8] V. E. Tarasov, Fractional Dynamics: Application of Fractional Calculus to Dynamics of Particles, Fields and Media, Springer, 2010.

[9] E. Set, "New inequalities of Ostrowski type for mappings whose derivatives are $s$-convex in the second sense via fractional integrals," Computers \& Mathematics with Applications, vol. 63, no. 7, pp. 1147-1154, 2012. 
[10] M. Z. Sarikaya, E. Set, H. Yaldiz, and N. Başak, "HermiteHadamard's inequalities for fractional integrals and related fractional inequalities," Mathematical and Computer Modelling, vol. 57, no. 9-10, pp. 2403-2407, 2013.

[11] C. Zhu, M. Fečkan, and J. Wang, "Fractional integral inequalities for differentiable convex mappings and applications to special means and a midpoint formula," Journal of Applied Mathematics, Statistics and Informatics, vol. 8, pp. 21-28, 2012.

[12] J. Wang, X. Li, and C. Zhu, "Refinements of hermiteHADamard type inequalities involving fractional integrals," Bulletin of the Belgian Mathematical Society-Simon Stevin, vol. 20, no. 4, pp. 655-666, 2013.

[13] J. Wang, J. Deng, and M. Fečkan, "Hermite-hadamardtype inequalities for $r$-convex functions based on the use of Riemann-liouville fractional integrals," Ukrainian Mathematical Journal, vol. 65, no. 2, pp. 193-211, 2013.

[14] Y. Zhang and J. Wang, "On some new Hermite-HADamard inequalities involving Riemann-Liouville fractional integrals," Journal of Inequalities and Applications, vol. 2013, article 220, 2013.

[15] J. Wang, X. Li, and M. Fe.kan, "Hermite-Hadamard-type inequalities for Riemann-Liouville fractional integrals via two kinds of convexity," Applicable Analysis, vol. 92, no. 11, pp. 22412253, 2013.

[16] J. Wang, J. Deng, and M. Fečkan, "Exploring s-e-condition and applications to some Ostrowski type inequalities via Hadamard fractional integrals," Mathematica Slovaca. In press.

[17] J. Wang, C. Zhu, and Y. Zhou, "New generalized HermiteHADamard type inequalities and applications to special means," Journal of Inequalities and Applications, vol. 2013, article 325, 2013.

[18] J. Deng and J. Wang, "Fractional Hermite-Hadamard inequalities for $(\alpha, m)$-logarithmically convex functions," Journal of Inequalities and Applications, vol. 364, pp. 1-11, 2013.

[19] Y. Liao, J. Deng, and J. Wang, "Riemann-Liouville fractional Hermite-HADamard inequalities. Part I: for once differentiable geometric-arithmetically s-convex functions," Journal of Inequalities and Applications, vol. 2013, 13 pages, 2013.

[20] H. M. Srivastava, Z. H. Zhang, and Y. D. Wu, "Some further refinements and extensions of the Hermite-Hadamard and Jensen inequalities in several variables," Mathematical and Computer Modelling, vol. 54, no. 11-12, pp. 2709-2717, 2011.

[21] C. P. Niculescu, "Convexity according to means," Mathematical Inequalities \& Applications, vol. 6, no. 4, pp. 571-579, 2003.

[22] R. A. Satnoianu, "Improved GA-convexity inequalities," Journal of Inequalities in Pure and Applied Mathematics, vol. 3, article 82, 6 pages, 2002.

[23] C. E. M. Pearce and J. Pečarić, "Inequalities for differentiable mappings with application to special means and quadrature formula," Applied Mathematics Letters, vol. 13, no. 2, pp. 51-55, 2000 . 


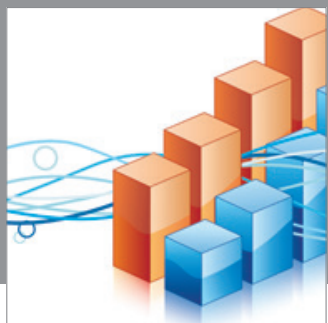

Advances in

Operations Research

mansans

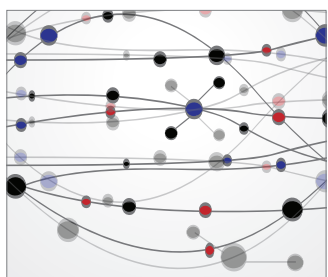

The Scientific World Journal
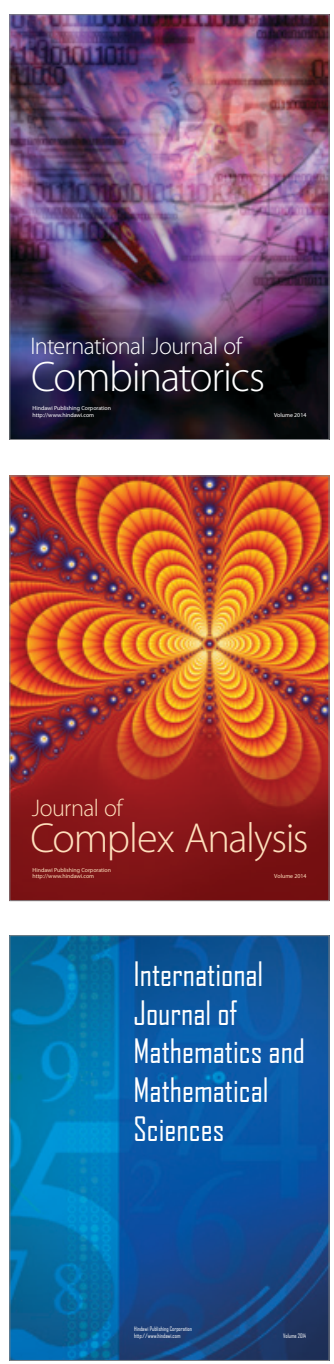
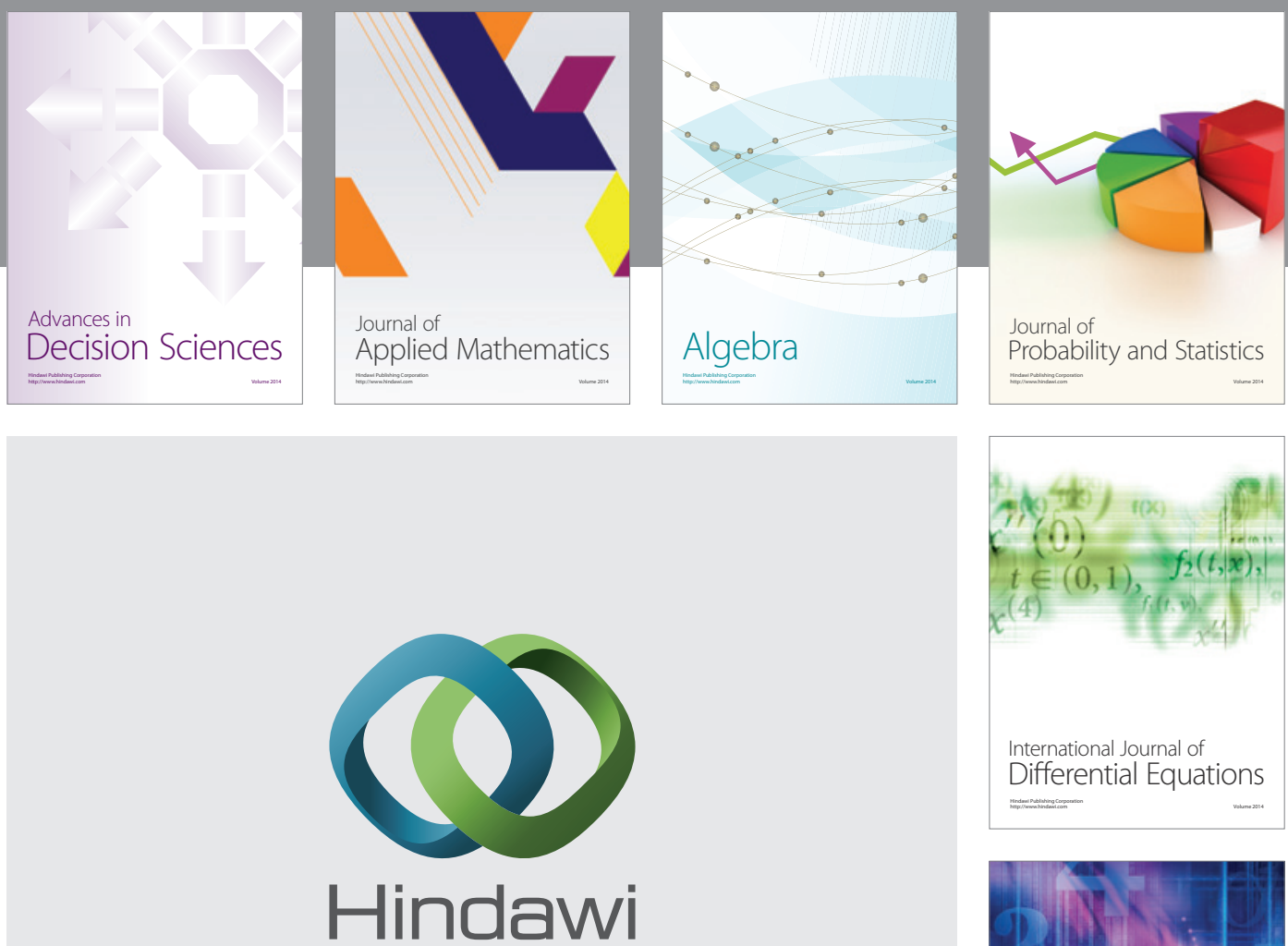

Submit your manuscripts at http://www.hindawi.com
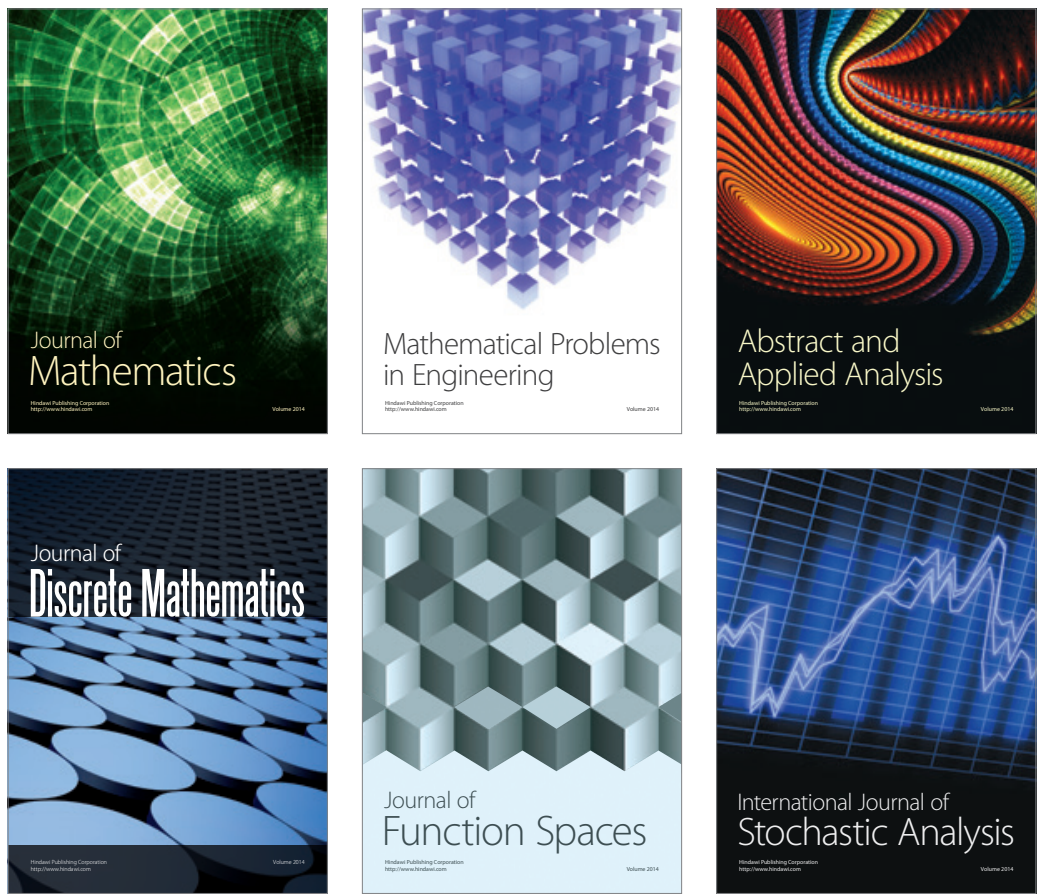

Journal of

Function Spaces

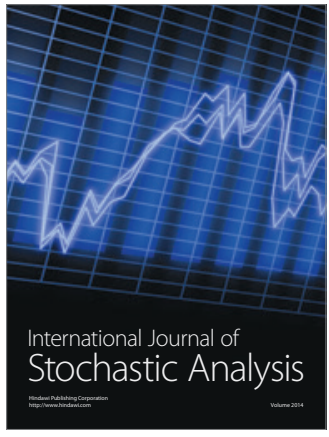

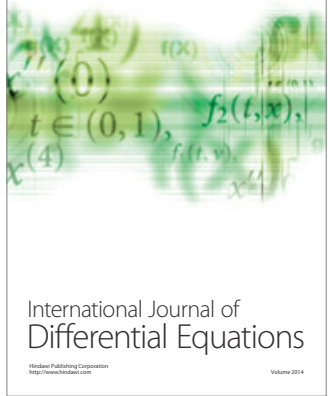
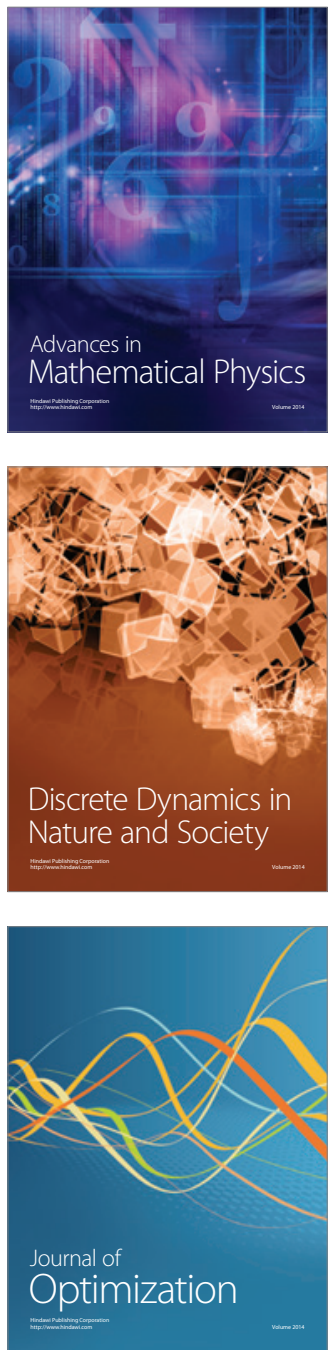